TRANSACTIONS OF THE

AMERICAN MATHEMATICAL SOCIETY

Volume 218,1976

\title{
MEAN CONVERGENCE OF FOURIER SERIES ON COMPACT LIE GROUPS
}

\author{
BY
}

ROBERT J. STANTON

\begin{abstract}
The main result is an $L^{p}$ mean convergence theorem for the partial sums of the Fourier series of a class function on a compact semisimple Lie group. A central element in the proof is a Lie group-Lie algebra analog of the theorems in classical Fourier analysis that allow one to pass back and forth between multiplier operators for Fourier series in several variables and multiplier operators for the Fourier transform in Euclidean space. To obtain the $L^{p}$ mean convergence theorem, the theory of the Hilbert transform with weight function is needed.
\end{abstract}

Introduction. A theorem of M. Riesz says that if $f$ is in $L^{p}$ of the circle, $1<p<\infty$, and if $S_{N} f(x)=\Sigma_{k=-N}^{N} a_{k} e^{i k x}$ is the $N$ th partial sum of the Fourier series of $f$, then $S_{N} f$ converges to $f$ in the $L^{p}$ norm as $N \rightarrow \infty$. Pollard [15] proved a similar result for Jacobi polynomials on the interval $[-1,1]$. If $f$ is in $L^{p}\left([-1,1] ;(1-x)^{\alpha}(1-x)^{\beta} d x\right)$ and if

$$
S_{N} f(x)=\sum_{k=0}^{N} d_{k}^{\alpha, \beta} a_{k} R_{k}^{\alpha, \beta}(x)
$$

is the $N$ th partial sum of the Jacobi series of $f$, then $S_{N} f$ converges to $f$ in the $L^{p}$ norm provided

$$
4 \max \left\{\frac{\alpha+1}{2 \alpha+3}, \frac{\beta+1}{2 \beta+3}\right\}<p<4 \min \left\{\frac{\alpha+1}{2 \alpha+1}, \frac{\beta+1}{2 \beta+1}\right\} .
$$

Here $R_{k}^{\alpha, \beta}$ is a normalized Jacobi polynomial and $d_{k}^{\alpha, \beta}$ is an appropriate constant.

It is well known that for suitable choices of $\alpha, \beta$ the $\left\{R_{k}^{\alpha, \beta}\right\}$ are the elementary spherical functions for the rank 1 symmetric spaces of compact type. Cast in this setting Pollard's theorem is an $L^{p}$ mean convergence result for bi- $K$ invariant functions on the rank 1 compact symmetric space $U / K$. In this paper we investigate extending this result to higher rank symmetric spaces.

Even in the abelian case of the $n$-torus $T^{n}$ the result depends drastically on how the multiple series is summed. Consider for example $f$ in $L^{p}\left(T^{n}\right)$ with

Received by the editors October 3, 1974 and, in revised form, March 12, 1975.

AMS (MOS) subject classifications (1970). Primary 43A90, 43A75; Secondary 33A45, $33 \mathrm{A75}$. 
Fourier series $\Sigma_{k=\left(k_{1}, \ldots, k_{n}\right)} a_{k} e^{i k \cdot x}$ and form the spherical partial sums

$$
S_{R} f(x)=\sum_{k_{1}^{2}+\ldots+k_{n}^{2} \leqslant R^{2}} a_{k} e^{i k \cdot x} .
$$

Fefferman [6] has proved that $S_{R} f$ converges to $f$ in the $L^{p}$ norm only if $p=2$. However if one uses square partial sums

$$
S_{N} f(x)=\sum_{\left|k_{i}\right|<N} a_{k} e^{i k \cdot x}
$$

the problem essentially factors into $n$ iterates of the dimension 1 case and $L^{p}$ mean convergence holds for $1<p<\infty$.

Our main result is an $L^{p}$ mean convergence theorem for Fourier series of class functions on a compact semisimple Lie group in which the partial sums are analogous to the square ones for the torus. The method of proof closely parallels the abelian case. The technique is to transfer spherical multiplier problems on a general compact symmetric space (of which a compact semisimple Lie group is the the special case $G \times G / \Delta$ with $\Delta$ the diagonal subgroup) to equivalent Fourier multiplier problems on the tangent space at the identity coset of the symmetric space. For symmetric spaces that are Lie groups this equivalence is essentially established; however, for a general compact symmetric space we prove only one direction. The Fourier multiplier problems arising from a convex polyhedra method of summing the Fourier series of class functions turn out to reduce to problems about iterates of the Hilbert transform in one variable with weight functions. Results in [12] on the Hilbert transform with weight then provide the tool to prove these are bounded operators.

In $\S 1$ we summarize notation and review basic aspects of harmonic analysis on compact symmetric spaces and of Fourier analysis on the tangent space at the identity coset.

In $\S 2$ passage from spherical multipliers on the symmetric space to Fourier multipliers on the tangent space is obtained.

From $\S 3$ on, we restrict attention to compact semisimple Lie groups. In $\S 3$ we prove a multiplier restriction theorem from Fourier multipliers on invariant functions to spherical multipliers on class functions. Then for these multipliers the problem on the group and the problem on the Lie algebra are equivalent. An observation further reduces Fourier multiplier problems on invariant functions on the Lie algebra to classical Fourier multipliers on Euclidean space with weight functions.

In $\S 4$ we apply the results of the preceding sections to prove the mean convergence theorem for Fourier series of class functions. In the converse direction, we establish continuity properties of the Fourier transform of invariant functions on the Lie algebra that yield negative results for multiplier problems.

I would like to thank my thesis advisor, A. W. Knapp, for his invaluable 
advice and encouragement while this research was being done. It is also a pleasure to thank R. Coifman, C. Fefferman, E. M. Stein, R. Strichartz and P. Tomas for their suggestions.

1. Fourier transforms, multipliers, and Bessel functions. Let $U$ be a compact simply connected semisimple Lie group. Let $\tilde{\theta}$ be an involution on $U$ and $K$ its fixed point set. $K$ is a connected compact subgroup and $U / K$ is a Riemannian globally symmetric space of compact type which is also simply connected. Let $u$ and $\mathfrak{t}$ be the respective Lie algebras. Then $\tilde{\theta}$ induces an involution automorphism $\theta$ of $u$ and a direct sum decomposition $u=\notin \oplus p_{*}$ where $E$ (resp. $p_{*}$ ) is the +1 (resp. -1 ) eigenspace of $\theta$. The concept of roots and restricted roots can be defined intrinsically for $U$ and $U / K$; however, we shall define them by means of the noncompact duals since we shall have to introduce these duals later anyway.

Let $g^{\mathbf{C}}$ be the complexification of $u$ and extend $\theta$ to $g \mathbf{C}$. Set $p=i p_{*}$ and $g_{0}=\xi \oplus p$. Then $g_{0}$ is a noncompact semisimple Lie algebra with Cartan involution $\theta$. Let $a$ be a maximal abelian subspace of $p$ and $\bar{G}$ a $\theta$-stable Cartan subalgebra of $g_{0}$ containing $a$. Then $\mathfrak{G}=\mathfrak{G}_{t} \oplus a$ where $\mathfrak{G}_{t}=\mathscr{G} \cap \mathfrak{E}$. The complexification $\mathfrak{G}^{\mathbf{C}}$ of $\mathfrak{G}$ is a Cartan subalgebra of $g^{C}$, and we denote the set of roots of $g^{\mathbf{C}}$ with respect to $\mathfrak{G}^{\mathbf{C}}$ by $\Delta$. We fix compatible orderings on the real duals of $a$ and $\mathfrak{G}_{R}=a \oplus i \mathfrak{G}_{\mathfrak{k}}$. Since the roots take real values in $\mathfrak{G}_{R}$ we get an order on $\Delta$. Denote by $\Delta^{+}$the set of positive roots and $G_{R}^{+}$the corresponding positive Weyl chamber.

The Killing form $B(\cdot, \cdot)$ of $g^{\text {C }}$ restricted to $\mathscr{G}_{R} \times \mathscr{G}_{R}$ is nondegenerate and we use $B$ to identify $\mathscr{G}_{R}$ and $\mathfrak{G}_{R}^{\prime}$ in the usual way. For $\lambda, \mu$ in $\mathfrak{G}_{R}^{\prime}$ we denote by $\langle\lambda, \mu\rangle$ the bilinear form induced by $B$. An element $\lambda$ in $\zeta_{R}^{\prime}$ is called integral if for all $\alpha \in \Delta, 2\langle\lambda, \alpha\rangle\langle\alpha, \alpha\rangle \in Z$ and is called dominant if for all $\alpha \in$ $\Delta^{+}, 2\langle\lambda, \alpha\rangle\left\langle\langle\alpha, \alpha\rangle \geqslant 0\right.$. Let $L \subset \mathscr{G}_{R}^{\prime}$ denote the semilattice of dominant integral linear functionals. The set of equivalence classes of irreducible unitary representations of $U$ is in $1-1$ correspondence with $L$. The semilattice in $\xi_{R}$ dual to $L$ by means of $B$ is contained in the closure of $\mathscr{G}_{R}^{+}$.

Set $P_{+}=\left\{\alpha \in \Delta^{+} \mid \alpha(H) \neq \alpha(\theta H)\right.$ for some $\left.H \in \mathfrak{G}_{R}\right\}$ and set $a^{+}=$ $\left\{H \in a \mid \alpha(H)>0\right.$ for all $\left.\alpha \in P_{+}\right\}$. Let $M^{\prime}$ be the normalizer of $a$ in $K$ and $M$ the centralizer of $a$ in $K$. The Weyl group $W=M^{\prime} / M$ leaves $a$ invariant and has $a^{+}$for a fundamental domain.

We now review some aspects of analysis on $U / K$, most of which can be found in Helgason [9] or Coifman and Weiss [3]. Let $d u$ and $d k$ denote Haar measures on $U$ and $K$ normalized to have total mass 1 and let $d \dot{u}$ denote the $U$ invariant measure on $U / K$ with total mass 1 . Let $L^{2}(U / K)$ be the Hilbert space of square integrable measurable functions on $U / K$. 
The left regular representation of $U$ on $L^{2}(U / K)$ provides a decomposition of $L^{2}(U / K)$ into an orthogonal direct sum of minimal invariant subspaces. The irreducible subrepresentations are precisely the class 1 representations of $(U, K)$, those with a one-dimensional subspace of $K$-fixed vectors, and occur with multiplicity 1. Let $\Lambda \subset L$ be the set of class 1 highest weights. For $\lambda$ in $\Lambda$ let $V_{\lambda}$ be the subspace of $L^{2}(U / K)$ of $\lambda$ type, let $d_{\lambda}=\operatorname{dim} V_{\lambda}$ and let $\varphi_{\lambda}$ in $V_{\lambda}$ be the corresponding elementary spherical function. The dual of $\Lambda$ is contained in $\overline{a^{+}}$.

The projection operator $P_{\lambda}: L^{2}(U / K) \rightarrow V_{\lambda}$ may be realized as an integral operator in the following way. If $f$ is in $L^{2}(U / K)$ and the projection $U \rightarrow U / K$ is denoted by $u \rightarrow \dot{u}$ then

$$
P_{\lambda} f(x)=d_{\lambda} \int_{U} f(\dot{u}) \varphi_{\lambda}\left(u^{-1} x\right) d u, \quad x \in U / K .
$$

We denote this integral by $P_{\lambda} f=d_{\lambda} f * \varphi_{\lambda}$.

For $f$ in $L^{2}(U / K)$ we call the expression $\Sigma_{\lambda \in \Lambda} d_{\lambda} f * \varphi_{\lambda}$ the Fourier series of $f$. If $f$ is in $L^{2}(K \backslash U / K)$ the Fourier series of $f$ has the simpler form $\Sigma_{\lambda \in \Lambda} d_{\lambda} \hat{f}(\lambda) \varphi_{\lambda}$ where $\hat{f}(\lambda)=\int_{U / K} f(\dot{u}) \overline{\varphi_{\lambda}(\dot{u})} d \dot{u}$.

A bounded linear operator $T$ on $L^{p}(U / K)$ is called a spherical multiplier operator if there exists a sequence $\{m(\lambda)\}_{\lambda \in \Lambda}$ such that for $f$ in $L^{2}(U / K) \cap$ $L^{p}(U / K), T f=\Sigma_{\lambda \in \Lambda} m(\lambda) d_{\lambda} f * \varphi_{\lambda}$. Spherical multiplier operators on $L^{p}(K \backslash U / K)$ are defined similarly. In either case the sequence is called a spherical multiplier. Spherical multiplier operators on $L^{p}(U / K)$ can be characterized as bounded linear operators commuting with left translation by $K$.

The Riemannian structure on $U / K$ that we have implicitly assumed is the restriction of $B$ to $p_{*} \times p_{*}$ and then translated by $U$. The fact that $B$ restricted to $\mathfrak{p}_{*} \times p_{*}$ is negative definite will now be exploited to study Fourier analysis on $p_{*}$.

We view $b_{*}$ as a locally compact abelian group self-dual by means of $B$. Lebesgue measure $d X$ on $p_{*}$ is normalized to be self-dual. For $f, g$ in $C_{c}\left(p_{*}\right)$ the Fourier transform is given by

$$
\hat{f}(Y)=\int_{\mathfrak{p}_{*}} f(X) e^{-i B(X, Y)} d X, \quad Y \in \mathfrak{p}_{*},
$$

and the Fourier inversion formula by

$$
\check{g}(X)=\int_{D_{*}} g(Y) e^{i B(X, Y)} d Y .
$$

A bounded linear operator $T$ on $L^{p}\left(p_{*}\right)$ is called a multiplier operator if there exists a measurable function $m$ so that $T f=[m \hat{f}]^{\sim}$ for $f$ in $L^{2}\left(p_{*}\right) \cap L^{p}\left(p_{*}\right)$.

The group $K$ acts on $p_{*}$ via the adjoint map. Set $a_{*}^{+}=-i a^{+}$. The map $\Phi: K / M \times a_{*}^{+} \rightarrow p_{*}$ given by $\Phi(k M, H)=\operatorname{Ad} k(H)$ is a diffeomorphism onto a dense open subset. A measurable function $f$ on $p_{*}$ is called Ad $K$ invariant if for 
all $k$ in $K$ and almost all $X$ in $p_{*}, f(\operatorname{Ad} k(X))=f(X)$. By the above diffeomorphism such a function is specified a.e. by its values on $a_{*}^{+}$. Corresponding to $\Phi$ there is an integration formula:

$$
\int_{\mathfrak{p}_{*}} f(X) d X=\int_{a_{*}^{+}} \int_{K} f(\operatorname{Ad} k(H)) d k\left|\prod_{\alpha \in P_{+}} \alpha(H)\right| d H, \quad f \in C_{c}\left(\mathfrak{p}_{*}\right) .
$$

Now suppose $f$ is in $C_{c}\left(P_{*}\right)$ and is $\operatorname{Ad} K$ invariant. Then

$$
\begin{aligned}
\hat{f}(Y) & =\int_{\mathfrak{q}_{*}} f(X) e^{-i B(X, Y)} d X \\
& =\int_{a_{*}^{+}} \int_{K} f(\operatorname{Ad} k(H)) e^{-i B(\operatorname{Ad} k(H), Y)}\left|\prod_{\alpha \in P_{+}} \alpha(H)\right| d k d H \\
& =\int_{a_{*}^{+}} f(H)\left[\int_{K} e^{-i B(\operatorname{Ad} k(H), Y)} d k\right]\left|\prod_{\alpha \in P_{+}} \alpha(H)\right| d H .
\end{aligned}
$$

From this we see that $\hat{f}$ is also Ad $K$ invariant. We define a function $J$ on $\overline{a_{*}^{+}} \times$ $\overline{a_{*}^{+}}$by

$$
J\left(H_{1}, H_{2}\right)=\int_{K} e^{i B\left(\operatorname{Ad} k\left(H_{1}\right), H_{2}\right)} d k .
$$

$J$ is $W$ invariant and so extends to an $\operatorname{Ad} K$ invariant function on $p_{*} \times p_{*}$. We call $J$ a Bessel function since for rank $U / K=1$ these are classical Bessel functions $J_{k}(x) /|x|^{k}$. These functions have a very rich structure and many properties in common with the classical Bessel functions. The proofs of the properties are group theoretic or geometric in nature. In the next section one of these properties is developed that has applications to multiplier problems.

2. A multiplier theorem from confluence. In this section we derive a relationship between spherical functions on the symmetric space and Bessel functions on $p_{*}$ considered as the tangent space at the identity coset of the symmetric space. This is a generalization of the classical Mehler-Heine theorem relating Jacobi polynomials and Bessel functions. We then take the first step toward transferring spherical multiplier problems on $U / K$ to equivalent Fourier multiplier problems on $p_{*}$. We prove that an $\operatorname{Ad} K$ invariant function is a Fourier multiplier on $b_{*}$ if a certain family of sequences obtained from this function are uniformly bounded spherical multipliers on $U / K$.

Let $G^{\mathrm{C}}$ be the complexification of $U$ and $G_{0}$ the real analytic subgroup of ${ }_{G} \mathbf{C}$ with Lie algebra $g_{0}$. Corresponding to a we have a local Iwasawa decomposition $g_{0}=k \oplus a \oplus n$ and a global Iwasawa decomposition $G_{0}=K A N$.

LEMma 2.1. (a) There exist neighborhoods $N_{0}$ of 0 in $g^{\mathrm{C}}$ and $N_{e}$ of $e$ in $G^{\mathrm{C}}$ such that exp: $N_{0} \rightarrow N_{e}$ is a complex biholomorphic map.

(b) Suppose $g^{C}=L_{1} \oplus L_{2} \oplus L_{3}$ with the $L_{i}$ complex subspaces. Let $V$ be an open neighborhood of 0 in $g^{C}$ such that if $Y$ is in $V$ and $Y=X_{1}+X_{2}+$ 
$X_{3}$ with $X_{i} \in L_{i}$, then $\exp X_{1} \exp X_{2} \exp X_{3}$ is in $N_{e}$. Then the map $\varphi: V \rightarrow$ $N_{0}$ given by $\varphi(Y)=\log \left(\exp X_{1} \exp X_{2} \exp X_{3}\right)$ has $d \varphi(0)$ equal to the identity and is a complex biholomorphic map of an open neighborhood of 0 in $\mathrm{g} \mathbf{C}$ onto another such neighborhood.

Proof. This is essentially the existence of holomorphic canonical coordinates of the 1st and 2nd kind and the proof in Hochschild [11] for real Lie groups can be repeated for the complex case.

We apply Lemma 2.1 to the complexified local Iwasawa decomposition. Write $g^{\mathbf{C}}=\mathfrak{E}^{\mathbf{C}} \oplus a^{\mathbf{C}} \oplus \mathrm{n}^{\mathrm{C}}$ and for $Z$ in $g^{\mathbf{C}}$ let $\mathcal{S}_{2}(Z)$ be the projection of $Z$ onto $a^{\mathrm{C}}$. For $g$ in $G_{0}$ let $H(g)$ in a be defined by $g=k \exp H(g) n$. We want to continue $H$ analytically to a neighborhood of $e$ in $G^{\mathrm{C}}$. Let $V_{1}$ and $V_{2}$ be the neighborhoods of 0 given by Lemma 2.1(b). The map $\sqrt{2} \circ \varphi^{-1} \circ \exp ^{-1}$ is holomorphic from $\exp V_{1}$ to $a^{\mathrm{C}}$ and agrees with $H$ on $\exp V_{1} \cap G_{0}$. We denote this extension of $H$ also by $H$.

LEMma 2.2. There exists a neighborhood $V$ of 0 in $g^{\mathbf{C}}$ and a positive number $\epsilon$ such that

$$
H(\exp t Z)=t \$(Z)+O\left(t^{2}\right)
$$

for $Z$ in $V$ and $t \in \mathrm{C}$ with $|t|<\epsilon$.

Proof. We use a series expansion for $H$ and Lemma 2.1. Let $\tau$ denote conjugation on $g^{\mathrm{C}}$ with respect to $u$. Then $B_{\tau}(X, Y)=-B(X, \tau Y)$ is a positive definite hermitian inner product on $g^{\mathbf{C}}$. For $Z$ in $g^{\mathbf{C}}$ set $\|Z\|^{2}=B_{\tau}(Z, Z)$. Now choose $V_{1}$ as above. As $H$ is holomorphic on $V_{1}$ for some $\epsilon>0$, the ball $B_{\epsilon}=\left\{Z \in g^{C} \mid\|Z\|<\epsilon\right\}$ is contained in $V_{1}$ and $H$ has a series expansion at $e$ valid on $\exp B_{\epsilon}$. So for $Z$ in $B_{\epsilon}$ and $t \in \mathrm{C}$ with $|t|<1, t Z$ is in $B_{\epsilon}$ and

$$
H(\exp t Z)=H(e)+d H(e)(t Z)+O\left(t^{2}\right) .
$$

We shall denote the point at which the differential is computed by a subscript on the differential. $H(e)=0$ and on $B_{\epsilon}$ exp defines a chart, so that

$$
H(\exp t Z)=d(H \circ \exp )_{0}(t Z)+O\left(t^{2}\right) .
$$

Since $H \circ \exp =\mathscr{F}_{2} \circ \varphi^{-1}, d(H \circ \exp )_{0}=d \mathscr{F}_{\varphi^{-1}(0)} \circ d \varphi_{0}^{-1}$. By Lemma 2.1 $d \varphi_{0}^{-1}$ is equal to the identity; $\mathscr{F}$ linear means that $d \mathscr{T}=\mathcal{S}_{2}$. Then

$$
d(H \circ \exp )_{0}(t Z)=t \mathscr{S}(Z),
$$

and we have the desired result.

Harish-Chandra [8] obtained an integral formula for spherical functions corresponding to finite-dimensional class 1 representations of $G_{0}:$ If $\lambda$ is in $\Lambda$, then 


$$
\varphi_{\lambda}(x)=\int_{K} e^{\lambda(H(x k))} d k \quad \text { for } x \in G_{0} .
$$

The analytic continuation of the $H$ function allows us to obtain a local integral formula for $\varphi_{\lambda}$ on $U$. In fact,

$$
H(\exp X k)=H\left(k k^{-1} \exp X k\right)=H\left(\exp A d k^{-1}(X)\right) \text { for } X \in \mathrm{B}_{0} \text {, }
$$

and $B_{\epsilon}$ is invariant under $\mathrm{Ad} K$; thus the integrand in Harich-Chandra's formula for $\varphi_{\lambda}$ is defined for $x=\exp Z$ with $Z$ in $B_{\epsilon}$. Now $\varphi_{\lambda}$ is a matrix coefficient of a finite-dimensional representation of $G^{\mathbf{C}}$, hence is holomorphic on all $G^{\mathbf{C}}$. On $\exp B_{\epsilon}, \varphi_{\lambda}$ and the analytically continued integral are holomorphic and agree on $\exp \left(B_{\epsilon}\right) \cap G_{0}$, hence are equal on $\exp B_{\epsilon}$. Summarizing, we have

Proposition 2.3. If $X$ is in $u$ and $\|X\|<\epsilon$, then

$$
\varphi_{\lambda}(\exp X)=\int_{K} e^{\lambda(H(\operatorname{expAd} k(X)))} d k .
$$

Remark. For special $U$, e.g., rank $U / K=1$, the $\epsilon$ can be determined and $\exp B_{\epsilon}$ fills a dense open subset of $U$. The integral has been called a "Laplace type" integral because for $S U(2) / S O(2)$ it gives the Laplace integral for Legendre polynomials.

Proposition 2.4. For $\lambda \in \Lambda$ and $n$ a positive integer

$$
\lim _{n \rightarrow \infty} \varphi_{n \lambda}(\exp X / n)=J\left(H_{\lambda}^{*}, X\right), \quad X \in u,
$$

with the convergence uniform on compact subsets of $u$.

Proof. Recall from $\S 1$ that the element dual to $\lambda$ is in $\overline{a^{+}}$. If $H_{\lambda}$ is this element, we set $H_{\lambda}^{*}=-i H_{\lambda}$. Then $H_{\lambda}^{*}$ is in $a_{*}^{+}$. Let $L \subset u$ be a compact set. Then $L$ is contained in $B_{N}$, a ball of radius $N$, for $N$ large enough. Let $\epsilon$ be as in Proposition 2.3. For all $n>N / \epsilon$ and for all $X$ in $L, X / n$ is in $B_{\epsilon}$. Then

$$
\varphi_{n \lambda}\left(\exp \frac{X}{n}\right)=\int_{K} e^{n \lambda(H(\exp \operatorname{Ad} k(X / n)))} d k
$$

By Lemma 2.2 we have

$$
\begin{aligned}
& \varphi_{n \lambda}\left(\exp \frac{X}{n}\right)=\int_{K} e^{n \lambda(\$(\operatorname{Ad} k(X / n)))} e^{n \lambda\left(O\left(1 / n^{2}\right)\right)} d k \\
& =\int_{K} e^{\lambda(\$(\operatorname{Ad} k(X)))} e^{n \lambda\left(O\left(1 / n^{2}\right)\right)} d k .
\end{aligned}
$$

As $n \rightarrow \infty, n \lambda\left(O\left(1 / n^{2}\right)\right) \rightarrow 0$, so that $e^{n \lambda\left(O\left(1 / n^{2}\right)\right)} \rightarrow 1$. Then

Recall that

$$
\lim _{n \rightarrow \infty} \varphi_{n \lambda}\left(\exp \frac{X}{n}\right)=\int_{K} e^{\lambda(\mathfrak{S}(\operatorname{Ad} k(X)))} d k .
$$

$$
J\left(H_{\lambda}^{*}, X\right)=\int_{K} e^{i B\left(H_{\lambda}^{*}, \operatorname{Ad} k(X)\right)} d k
$$


To complete the proof we need only observe that

$$
B\left(H_{\lambda}, \operatorname{Ad} k(X)\right)=B\left(H_{\lambda}, \mathfrak{Q}(\operatorname{Ad} k(X))\right)=\lambda(\$(\operatorname{Ad} k(X))) .
$$

To see the first equality suppose $Z$ is in $3^{\mathrm{C}}$ and $Z=X_{1}+X_{2}+X_{3}$ with $X_{1} \in$ $\mathfrak{E}^{\mathrm{C}}, X_{2}=\mathcal{F}_{2}(Z), X_{3} \in \mathrm{n}^{\mathrm{C}}$. Then $\mathfrak{k} \perp$ implies that $B\left(H_{\lambda}, X_{1}\right)=0$. Lemma VI, 3.5 in Helgason [9] shows the existence of a basis of $g^{\mathbf{C}}$ with respect to which ad $X_{3}$ is strictly lower triangular and ad $H_{\lambda}$ is diagonal. Hence $B\left(H_{\lambda}, X_{3}\right)$ $=0$.

THEOREM 2.5. Let $m$ be a bounded Ad $K$ invariant function on $p_{*}$ that is continuous a.e. Suppose that for each $r>0$ the sequence $\left\{m\left(H_{\lambda}^{*} / r\right)\right\}_{\lambda \in \Lambda}$ is a spherical multiplier on $L^{p}(U / K)$ and the norms of the corresponding operators are uniformly bounded. Then $m$ is a multiplier on $L^{p}\left(p_{*}\right)(1<p<\infty)$.

REMARK. This result was proved by Igari [13] and Clerc [2] independently when $\operatorname{rank} U / K=1$. Strichartz [18] then extended it to symmetric spaces that are compact Lie groups. Proposition 2.4 allows us to push Strichartz's proof through for arbitrary compact $U / K$. Clerc has independently found Proposition 2.4 with essentially the same proof. We include the proof then only for completeness.

Proof. Let $q$ be the index conjugate to $p$. We first assume $m$ decreases rapidly, say $|m(X)| \leqslant c_{1} \exp \left(-c_{2}\|X\|^{2}\right)$. Let $f$ and $g$ be in $C_{c}^{\infty}\left(p_{*}\right)$. It suffices to show that $I=\int_{R_{*}} T f(X) g(X) d X$ is bounded in absolute value by $C\|f\|_{p}\|g\|_{q}$ with $C$ independent of $f$ and $g$. The idea is to obtain a Riemann sum approximation to $I$ by using Proposition 2.4 and then to use the uniform boundedness of the operators corresponding to the dilates of $m$.

$$
\begin{aligned}
I & =\int_{\mathfrak{p}_{*}} T f(X) g(X) d X=\int_{\mathfrak{p}_{*}} \int_{\mathfrak{p}_{*}} m(Z) \hat{f}(Z) e^{i B(X, Z)} d Z g(X) d X \\
& =\int_{\mathfrak{p}_{*}} \int_{\mathfrak{p}_{*}} \int_{\mathfrak{p}_{*}} m(z) e^{i B(X, Z)} f(Y) e^{-i B(Y, Z)} g(X) d Y d Z d X .
\end{aligned}
$$

As $m$ decreases rapidly, we may apply the Fubini theorem to the two inside integrals and then use the integration formula for $\mathrm{Ad} K$ invariant functions on $p_{*}$ to obtain

$$
I=\int_{\mathfrak{p}_{*}} \int_{\mathfrak{p}_{*}} \int_{a_{*}} m(H) J(H, X-Y)\left|\prod_{\alpha \in P_{+}} \alpha(H)\right| d H f(Y) g(X) d Y d X .
$$

Now choose $R$ large enough so that exp is a chart on $R^{-1}$ supp $f$ and $R^{-1}$ supp $g$. Define functions $f_{R}$ and $g_{R}$ on $U / K$ by $f_{R}(\exp X / R)=f(X)$ and $g_{R}(\exp X / R)=$ $g(X)$. Let $T_{R}$ be the operator corresponding to $\left\{m\left(H_{\lambda}^{*} / R\right)\right\}_{\lambda \in \Lambda}$ and let $I_{R}=$ $\int_{U / K} T_{R} f_{R}(\dot{u}) g_{R}(\dot{u}) d \dot{u}$. By hypothesis there exists $A<\infty$ independent of $R, f, g$ such that $\left|I_{R}\right| \leqslant A\left\|f_{R}\right\|_{p}\left\|g_{R}\right\|_{q}$. We claim there is a constant $C$ depend- 
ing only on $U$ such that $R^{N} I_{R} \rightarrow C I$ as $R \rightarrow \infty$ where $N=\operatorname{dim} U / K$. In fact

$$
\begin{array}{r}
R^{N} I_{R}=R^{N} \int_{U / K} \int_{U / K} \sum_{\lambda \in \Lambda} m\left(\frac{H_{\lambda}^{*}}{R}\right) d_{\lambda} \varphi_{\lambda}\left(y^{-1} x\right) f_{R}(y) g_{R}(x) d \dot{u}(y) d \dot{u}(x), \\
R^{N} I_{R}=R^{N} \int_{D_{*}} \int_{D_{*}} \sum_{\lambda \in \Lambda} m\left(\frac{H_{\lambda}^{*}}{R}\right) d_{\lambda} \varphi_{\lambda}(\exp -Y \exp X) \\
\times f_{R}(\exp Y) g_{R}(\exp X) \Delta_{0}(Y) \Delta_{0}(X) d Y d X,
\end{array}
$$

where we have used exp as a chart and if $Y=\operatorname{Ad} k H$,

$$
\Delta_{0}(Y)=\text { Jacobian } \exp _{e K}(Y)=\Delta_{0}(\text { Ad } k H)=\prod_{\alpha \in P_{+}} \frac{\sin \alpha(i H)}{\alpha(i H)} .
$$

Now we make the change of variables $Y=Y / R, X=X / R$ to obtain

$$
R^{N} I_{R}=R^{N} \cdot R^{-2 N} \int_{\mathfrak{p}_{*}} \int_{\mathfrak{p}_{*}} \sum_{\lambda \in \Lambda} m\left(\frac{H_{\lambda}^{*}}{R}\right) d_{\lambda} \varphi_{\lambda}(\exp -Y / R \exp X / R)
$$

Consider the inside sum

$$
\times f(Y) f(X) \Delta_{0}(Y / R) \Delta_{0}(X / R) d Y d X
$$

$$
R^{-N} \sum_{\lambda \in \Lambda} m\left(H_{\lambda}^{*} / R\right) d_{\lambda} \varphi_{\lambda}(\exp -Y / R \exp X / R) \Delta_{0}(Y / R) \Delta_{0}(X / R) .
$$

As $R \rightarrow \infty, \Delta_{0}(Y / R)$ and $\Delta_{0}(X / R)$ tend to 1 and will be no problem. Using the Campbell-Hausdorff formula we see that $\exp -Y / R \exp X / R=\exp Z_{R} / R$ with $Z_{R} \rightarrow X-Y$ as $R \rightarrow \infty$. Make the change of variable $\lambda=\lambda / R$ so the expression becomes

$$
R^{-N} \sum_{\lambda \in \Lambda / R} m\left(H_{\lambda}^{*}\right) d_{\lambda R} \varphi_{R \lambda}\left(\exp Z_{R} / R\right) \Delta_{0}(Y / R) \Delta_{0}(X / R)
$$

Weyl's formula for the degree of a representation is

$$
d_{\lambda R}=\prod_{\alpha \in \Delta^{+}} \frac{\langle\alpha, \lambda R+\rho\rangle}{\langle\alpha, \rho\rangle} .
$$

As $\lambda R$ is class 1 we have $\langle\alpha, \lambda R\rangle=0$ for $\alpha \notin P_{+}$. If $\left|P_{+}\right|$is the cardinality of $P_{+}$, then

$$
d_{\lambda R}=R^{\mid P}+\mid \prod_{\alpha \in \Delta^{+}} \frac{\langle\alpha, \lambda+\rho / R\rangle}{\langle\alpha, \rho\rangle}
$$

and as $R \rightarrow \infty$,

$$
\frac{d_{\lambda R}}{R^{\mid P+1}} \rightarrow \frac{\Pi_{\alpha \in P_{+}}\langle\alpha, \lambda\rangle}{\Pi_{\alpha \in \Delta}+\langle\alpha, \rho\rangle}
$$

Since $N=\operatorname{dim} a_{*}+\left|P_{+}\right|$, the expression is

$$
\frac{1}{R^{\operatorname{dim} a_{*}}} \sum_{\lambda \in \Lambda / R} m\left(H_{\lambda}^{*}\right) \frac{d_{\lambda R}}{R^{\left|P_{+}\right|} \varphi_{R \lambda}}\left(\frac{\exp Z_{R}}{R}\right) \Delta_{0}\left(\frac{Y}{R}\right) \Delta_{0}\left(\frac{X}{R}\right) .
$$


Using Proposition 2.4 and the continuity a.e. of $m$ we see that the above is a Riemann sum approximation to

Then

$$
\frac{1}{\Pi_{\alpha \in \Delta^{+}}\langle\alpha, \rho\rangle} \int_{a_{*}^{+}} m(H) J(H, X-Y) \prod_{\alpha \in P_{+}}\langle\alpha, H\rangle d H .
$$

$$
\lim _{R \rightarrow \infty} \mathrm{R}^{N} I_{R}=\frac{1}{\Pi_{\alpha \in \Delta}+\langle\alpha, \rho\rangle} I
$$

which is the claim.

Finally,

$$
\begin{aligned}
|I| & =C \lim _{R \rightarrow \infty} R^{N}\left|\int_{U / K} T_{R} f_{R}(\dot{u}) g_{R}(\dot{u}) d \dot{u}\right|, \\
|I| & \leqslant C \lim _{R \rightarrow \infty} R^{N} \cdot A\left\|f_{R}\right\|_{p}\left\|g_{R}\right\|_{q} \\
& =C \cdot A \cdot \lim _{R \rightarrow \infty} R^{N / p}\left\|f_{R}\right\|_{p} R^{N / q}\left\|g_{R}\right\|_{q} \\
& =C \cdot A\|f\|_{p}\|g\|_{q} .
\end{aligned}
$$

And so $m$ is a multiplier on $L^{p}\left(p_{*}\right)$. To remove the hypothesis that $m$ decreases rapidly, define for $\epsilon>0$ a new multiplier $m^{\epsilon}(X) \exp \left(-\epsilon\|X\|^{2}\right)$ and observe that the constant $A$ can be chosen independent of $\epsilon$. Letting $\epsilon \rightarrow 0$, we have the desired result.

REMARK 2.6. Theorem 2.5 is true for multipliers on $\operatorname{Ad} K$ invariant functions on $p_{*}$ if the dilated multipliers are uniformly bounded operators on bi-K invariant functions on $U / K$. The proof is exactly the same. In fact if our original $f$ and $g$ were Ad $K$ invariant, the only fact one needs to check is that $f_{R}$ and $g_{R}$ are bi- $K$ invariant. But this is obvious.

REMARK 2.7. One can formulate Theorem 2.5 for weak type boundedness of multipliers rather than strong type. For rank 1, Connett and Schwartz [5] have proved such a result. Proposition 2.4 allows one to carry their proof over to arbitrary rank.

3. Restriction and transplantation of multipliers. When the rank of $U / K$ is 1 , one can use Theorem 2.5 to obtain many well-known multiplier theorems on $\mathbf{R}^{n}$ from the corresponding results for Jacobi polynomials (see Igari [13]). However, for higher rank little is known about analysis on $U / K$, whereas multiplier theorems on $\mathbf{R}^{n}$ have been deeply investigated. So it would be much more useful to have a "restriction" theorem for multipliers from the tangent space to the symmetric space.

Let $G$ be a compact simply connected semisimple Lie group and let $g$ be 
its Lie algebra. In this section we shall prove a theorem concerning restrictions of multipliers on $\operatorname{Ad} G$ invariant functions on $g$ to central multipliers on class functions on $G$. The analogous abelian theorem is due to de Leeuw [14], and our proof parallels the proof of de Leeuw's theorem found in Stein and Weiss [17]. Afterward we make an observation that allows us to transplant Fourier multiplier questions on $\mathrm{Ad} G$ invariant functions to classical Fourier multipliers on Euclidean space with weight functions. Coifman and Weiss [4] also have transplantation type results. The essential difference between their approach and ours is they use the weight function to modify the multiplier whereas we keep the multiplier fixed and face handling multiplier problems with weight.

The group $G$ with the Riemannian structure induced from the Killing form on $g$ is isometric to the symmetric space $U / K$ where $U=G \times G$ and $K=\Delta$, the diagonal subgroup. By means of this diffeomorphism, the bi- $K$ invariant func tions on $U$ corresponds to class functions on $G$ and the spherical functions on $U / K$ correspond to characters of irreducible unitary representations of $G$ divided by the degree of the representation. In this setting the restriction theorem in this section is a partial converse to Theorem 2.5 .

Since $G$ is a symmetric space the notation introduced in $\$ 1$ could still be used; however results in the literature that we need are expressed in more traditional notation. For the purpose of compatibility with the literature we employ the traditional notation.

Let $G$ be a compact simply connected semisimple Lie group and $T$ a maximal torus in $G$. Let $g$ and $\mathfrak{G}$ be their Lie algebras and $g^{C}$ and $h^{C}$ the respective complexifications. Let $\Delta$ be the set of roots of $\left(g^{\mathrm{C}}, b^{\mathrm{C}}\right) ; \Delta^{+}$the positive roots for some order; and $W$ the Weyl group. Let $\rho$ be half the sum of the positive roots. For $H$ in $\zeta^{\mathrm{C}}$ set

$$
\begin{aligned}
& D(H)=\prod_{\alpha \in \Delta^{+}}\left[e^{\alpha(H) / 2}-e^{-\alpha(H) / 2}\right], \\
& P(H)=\prod_{\alpha \in \Delta^{+}} \alpha(H) \text { and } D_{0}(H)=D(H) / P(H) .
\end{aligned}
$$

The positive roots determine a positive Weyl chamber $a \subseteq i \hbar$. Set $\zeta^{+}=-i a$. The Killing form on $g^{\mathbf{C}} \times g^{\mathbf{C}}$ will be denoted by $B(\cdot, \cdot)$. When restricted to $g \times g$ it agrees with the Killing form of $g$. The set of equivalence classes of irreducible unitary representations of $G$ are in 1-1 correspondence with the semi lattice $L$ of dominant integral forms. If $\lambda$ is in $L$, let $H_{\lambda}$ be its dual element in $i$ a and set $H_{\lambda}^{*}=-i H_{\lambda}$, let $\chi_{\lambda}$ be the character and $d_{\lambda}$ the degree of the associated representation. Normalization of measures is as in $\S 1$. If $\Gamma$ denotes $\{H \in \mathfrak{b}|| \alpha(H) \mid<2 \pi$ for all $\alpha \in \Delta\}$, then the Weyl integration formula for a class function $f$ is 


$$
\int_{G} f(g) d g=\frac{1}{|W|} \int_{\Gamma} f(\exp H)|D(H)|^{2} d H .
$$

The ability to transport analysis problems between group and algebra is provided by the following theorem of Harish-Chandra [7].

Proposition 3.1. For all $\mathrm{H}_{1}, \mathrm{H}_{2}$ in $\mathrm{G}^{\mathrm{C}}$

$$
P\left(H_{1}\right) P\left(H_{2}\right) \int_{G} e^{B\left(\operatorname{Ad} g\left(H_{1}\right), H_{2}\right)} d g=P\left(H_{\rho}^{*}\right) \sum_{s \in W} \epsilon(s) e^{B\left(s H_{1}, H_{2}\right)} .
$$

We set

$$
J\left(H_{1}, H_{2}\right)=\int_{G} e^{i B\left(\operatorname{Ad} g\left(H_{1}\right), H_{2}\right)} d g
$$

for $H_{1}, H_{2}$ in $\mathfrak{\xi}$. This function will be called a Bessel function. As it is $W$ invariant it has an extension to $g \times g$. The following properties of $J$ are easily verified.

(1) $J$ is Ad $G$ invariant in each variable, hence is specified by its values on $\overline{5+} \times \overline{5+}$.

(2) $J\left(H_{1}, H_{2}\right)=J\left(H_{2}, H_{1}\right) ; \overline{J\left(H_{1}, H_{2}\right)}=J\left(-H_{1}, H_{2}\right)=J\left(H_{1},-H_{2}\right)$.

(3) $\left|J\left(H_{1}, H_{2}\right)\right| \leqslant 1$ and $J\left(0, H_{2}\right)=1=J\left(H_{1}, 0\right)$.

(4) For $H_{1}, H_{2}$ in $\mathfrak{G}^{+}$,

$$
J\left(H_{1}, H_{2}\right)=\frac{P\left(H_{\rho}^{*}\right) \Sigma \epsilon(w) e^{i B\left(w H_{1}, H_{2}\right)}}{P\left(H_{1}\right) P\left(H_{2}\right)} .
$$

Proposition 3.2. For $\lambda$ in $L$ and $H$ in $5^{+}$

$$
J\left(H_{\lambda+\rho}^{*}, H\right)=D_{0}(H) \chi_{\lambda}(\exp H) / d_{\lambda} .
$$

Proof. Use the Weyl character formula, the Weyl dimension formula, and (4) above.

The Gauss kernel on $\mathfrak{b}$ is given by

$$
g_{s}(H)=c \exp \left(-\|\dot{H}\|^{2} / s\right) / s^{l / 2} \text { for } s>0 \text { and } l=\operatorname{dim} \zeta .
$$

Lemma 3.3. Let $\lambda$ be in $L$, regard $D_{0}$ and $g_{s}$ as extended to functions on $g$ and pull back $\chi_{\lambda}$ to an invariant function on $g$ via the exponential. Then for $H_{0} \in \mathfrak{G}^{+}$

$$
\begin{aligned}
{\left[D_{0} \frac{\chi_{\lambda}}{d_{\lambda}} g_{s}\right]^{\wedge} } & \left(H_{0}\right) \\
& =\frac{P\left(H_{\rho}^{*}\right)}{|W| d_{\lambda} P\left(H_{0}\right)} \sum_{W, W} \epsilon(\sigma) \epsilon(\tau) \exp \left(-s\left\|\tau H_{0}-\sigma H_{\lambda+\rho}^{*}\right\|^{2}\right) .
\end{aligned}
$$


Proof.

$$
\left[D_{0} \frac{\chi_{\lambda}}{d_{\lambda}} g_{s}\right]\left(H_{0}\right)=\frac{1}{|W|} \int_{G} D_{0}(H) \frac{\chi_{\lambda}(\exp H)}{d_{\lambda}} g_{s}(H) \overline{J\left(H, H_{0}\right)}|P(H)|^{2} d H .
$$

By Proposition 3.2 and the Weyl character formula the right side is

$$
\begin{aligned}
=\frac{1}{|W|} \int_{G} \frac{D(H)}{P(H)} \frac{\Sigma \epsilon(\sigma) e^{i B\left(\sigma H_{\lambda+\rho}^{*}, H\right)}}{d_{\lambda} D(H)} & \\
& \times \frac{P\left(H_{\rho}^{*}\right) \Sigma \epsilon(\tau) e^{-i B\left(\tau H_{0}, H\right)}}{P\left(H_{0}\right) P(H)} g_{s}(H)|P(H)|^{2} d H \\
= & \frac{P\left(H_{\rho}^{*}\right)}{|W| d_{\lambda} P\left(H_{0}\right)} \sum_{W, W} \epsilon(\sigma) \epsilon(\tau) \int_{G} g_{s}(H) e^{-i B\left(\tau H_{0}-\sigma H_{\sigma}^{*}+\rho^{\prime} H\right)} d H \\
= & \frac{P\left(H_{\rho}^{*}\right)}{|W| d_{\lambda} P\left(H_{0}\right)} \sum_{W, W} \epsilon(\sigma) \epsilon(\tau) \exp \left(-s\left\|\tau H_{0}-\sigma H_{\lambda+\rho}^{*}\right\|^{2}\right) .
\end{aligned}
$$

Next we shall develop a series of lemmas paralleling $\$ 3$ of Chapter 7 of Stein and Weiss [17].

Lemma 3.4. Let $f$ be a continuous class function. Then

$$
\lim _{s \rightarrow \infty} \int_{\zeta} g_{s}(H) f(\exp H)|D(H)|^{2} d H=\int_{\Gamma} f(\exp H)|D(H)|^{2} d H .
$$

Proof. This is a well-known fact in abelian harmonic analysis. A proof can be found in, e.g., [17].

Now suppose $m$ is a bounded measurable $\operatorname{Ad} G$ invariant function on $g$. Let $K$ be the multiplièr operator acting on $\operatorname{Ad} G$ invariant functions corresponding to $m$. If, in addition, $m$ is continuous at $H_{\lambda+\rho}^{*}$ for each $\lambda \in L$, we define a multiplier on $G$ by the sequence $m^{*}=\left\{m\left(H_{\lambda+\rho}^{*}\right)\right\}_{\lambda \in L}$. We denote by $K^{*}$ the corresponding operator on class functions on $G$. For the remainder of this section these hypotheses and notations will remain in effect.

LEMмA 3.5. For any characters $\chi_{\lambda}$ and $\chi_{\mu}$

$$
\begin{aligned}
\lim _{s \rightarrow \infty} s^{l / 2} \int_{\zeta} K & \left(D_{0} \frac{\chi_{\lambda}}{d_{\lambda}} g_{s}\right)(H) \overline{D_{0}(H)} \frac{\overline{\chi_{\mu}(\exp H)}}{d_{\mu}} g_{s}(H)|P(H)|^{2} d H \\
& =P\left(H_{\rho}^{*}\right)^{2} \int_{\Gamma} K^{*}\left(\frac{\chi_{\lambda}}{d_{\lambda}}\right)(\exp H) \frac{\overline{\chi_{\mu}(\exp H)}}{d_{\mu}}|D(H)|^{2} d H
\end{aligned}
$$


PROOF.

$$
\begin{aligned}
\left.s^{l / 2} \int_{G} K\left(D_{0} \frac{\chi_{\lambda}}{d_{\lambda}} g_{s}\right)(H) \overline{D_{0}(H)} \frac{\overline{\chi_{\mu}(\exp H)}}{d_{\mu}} g_{s}(H) i P(H)\right|^{2} d H \\
=s^{l / 2} \int_{\zeta} m(H)\left[D_{0} \frac{\chi_{\lambda}}{d_{\lambda}} g_{s}\right]^{\wedge}(H) \overline{\left[D_{0} \frac{\chi_{\mu}}{d_{\mu}} g_{s}\right]^{a}}(H)|P(H)|^{2} d H \\
=\frac{s^{l / 2} P\left(H_{\rho}^{*}\right)^{2}}{d_{\lambda} d_{\mu}} \int_{\varphi} m(H) \frac{\Sigma \epsilon\left(w_{1}\right) \exp \left(-s\left\|H-w_{1} H_{\lambda+\rho}^{*}\right\|^{2}\right)}{P(H)} \\
\times \frac{\Sigma \epsilon\left(w_{2}\right) \exp \left(-s\left\|H-w_{2} H_{\mu+\rho}^{*}\right\|^{2}\right)}{P(H)}|P(H)|^{2} d H \\
=\frac{P\left(H_{\rho}^{*}\right)^{2}}{d_{\lambda} d_{\mu}} s^{l / 2} \sum_{W, W} \epsilon\left(w_{1}\right) \epsilon\left(w_{2}\right) \int m(H) \exp \left(-s\left\|H-w_{1} H_{\lambda+\rho}^{*}\right\|^{2}\right) \\
\times \exp \left(-s\left\|H-w_{2} H_{\mu+\rho}\right\|^{2}\right) .
\end{aligned}
$$

If $\lambda \neq \mu$, the sets $\{\sigma(\lambda+\rho)\}$ and $\{\tau(\mu+\rho)\}$ are disjoint. So we choose $\delta$ small enough so that for all $w_{1}, w_{2}$ in $W,\left\|w_{1} H_{\lambda+\rho}^{*}-w_{2} H_{\mu+\rho}^{*}\right\|^{2}>2 \delta$. Then

$$
\begin{aligned}
& s^{l / 2} \mid \int_{G} m(H) \exp \left(-s\left\|H-w_{1} H_{\lambda+\rho}\right\|^{2} \exp \left(-s\left\|H-w_{2} H_{\mu+\rho}^{*}\right\|^{2}\right) d H \mid\right. \\
& \leqslant s^{l / 2} \int_{\left\|H-w_{1} H_{\lambda+\rho}^{*}\right\|^{2}>\delta}|m(H)| \exp \left(-s\left\|H-w_{2} H_{\mu+\rho}^{*}\right\|^{2}\right) e^{-\delta s} d H \\
& \quad+s^{l / 2} \int_{\left\|H-w_{2} H_{\mu+\rho}^{*}\right\|^{2}>\delta} \exp \left(-s\left\|H-w_{1} H_{\lambda+\rho}^{*}\right\|^{2}\right) e^{-\delta s}|m(H)| d H .
\end{aligned}
$$

And as $s \rightarrow \infty$ both terms on the right $\rightarrow 0$. Next we compute the integral on the group.

$$
\begin{aligned}
\int_{\Gamma} K^{*}\left(\frac{\chi_{\lambda}}{d_{\lambda}}\right)(\exp H) \frac{\overline{\chi_{\mu}(\exp H)}}{d_{\mu}}|D(H)|^{2} d H \\
=\frac{m\left(H_{\lambda+\rho}^{*}\right)}{d_{\lambda} d_{\mu}} \int_{\Gamma} \chi_{\lambda}(\exp H) \overline{\chi_{\mu}(\exp H)}|D(H)|^{2} d H=0 .
\end{aligned}
$$

Suppose then that $\lambda=\mu$. The argument above works for $w_{1}, w_{2}$ in $W$ with $w_{1} \neq w_{2}$ to show the integral on $\zeta$ is zero. If $w_{1}=w_{2}$,

$$
s^{l / 2} \frac{P\left(H_{P}^{*}\right)^{2}}{d_{\lambda}^{2}} \sum_{W} \int_{G} m(H) \exp \left(-2 s\left\|H-w H_{\lambda+\rho}^{*}\right\|^{2}\right) d H
$$

is the classical Gauss convolution on $\mathbf{R}^{l}$ and since $m$ is continuous at $H_{\lambda+\rho}^{*}$ it 
equals, in the limit $s \rightarrow \infty, P\left(H_{\rho}^{*}\right)^{2}|W| m\left(H_{\lambda+\rho}^{*}\right) / d_{\lambda}^{2}$. Again we compute the integral on the group and by orthonormality of characters we have

$$
\int_{\Gamma} K^{*}\left(\frac{\chi_{\lambda}}{d_{\lambda}}\right)(\exp H) \frac{\chi_{\lambda}(\exp H)}{d_{\lambda}}|D(H)|^{2} d H=|W| \frac{m\left(H_{\lambda+\rho}^{*}\right)}{d_{\lambda}^{2}}
$$

REMARK 3.6. The preceding lemma is true for trigonometric polynomials.

THEOREM 3.7. Suppose $m$ is an Ad $G$ invariant measurable function on $g$ that is continuous at $\left\{H_{\lambda+\rho}^{*} \mid \lambda \in L\right\}$. If there is a $C<\infty$ such that for $f$ in $L_{\text {inv }}^{p}(g)$

$$
\int_{\zeta}|K f(H)|^{p}\left|D_{0}(H)\right|^{2-p}|P(H)|^{2} d H \leqslant C \int_{6}|f(H)|^{p}\left|D_{0}(H)\right|^{2-p}|P(H)|^{2} d H,
$$

then $m^{*}=\left\{m\left(H_{\lambda+\rho}^{*}\right)\right\}_{\lambda \in L}$ is a multiplier on $L_{\mathrm{inv}}^{p}(G)$ with operator norm $\leqslant A C$ where $A$ depends only on $\mathrm{g}$.

Proof. We prove the theorem for $1<p<\infty$ and trigonometric polynomials. Suppose $f=\Sigma a_{\lambda} d_{\lambda} \chi_{\lambda}$ and $h=\Sigma b_{\lambda} d_{\lambda} \chi_{\lambda}$ are trigonometric polynomials. As usual it suffices to find a constant $A$ independent of $f, h$ with

$$
\left.\left|\int_{\Gamma} K^{*} f(\exp H) \overline{h(\exp H)}\right| D(H)\right|^{2} d H \mid \leqslant A\|f\|_{p}\|h\|_{q} .
$$

By Lemma 3.5,

$$
\begin{aligned}
I & =P\left(H_{\rho}^{*}\right)^{2} \int_{\Gamma} K^{*} f(\exp H) \overline{h(\exp H)}|D(H)|^{2} d H \\
& =\lim _{s \rightarrow \infty} s^{l / 2} \int_{\zeta} K\left(D_{0} g_{s} f\right)(H) \overline{D_{0}(H)} \overline{h(\exp H)} g_{s}(H)|P(H)|^{2} d H .
\end{aligned}
$$

Then

$$
|I| \leqslant \lim _{s \rightarrow \infty} s^{l / 2} \int_{\zeta}\left|K\left(D_{0} g_{s} f\right)(H)\right|\left|D_{0}(H) h(\exp H) g_{s}(H)\right||P(H)|^{2} d H .
$$

Writing $\left|D_{0}(H)\right|=\left|D_{0}(H)\right|^{1-2 / q}\left|D_{0}(H)\right|^{2 / q}$ and using Hölder's inequality we find that the right side

$$
\begin{aligned}
\leqslant & \lim _{s \rightarrow \infty} s^{l / 2}\left[\int_{\zeta}\left|K\left(D_{0} g_{s} f\right)(H)\right|^{p}\left|D_{0}(H)\right|^{2-p}|P(H)|^{2} d H\right]^{1 / p} \\
\cdot & {\left[\int_{\zeta}|h(H)|^{q}\left|g_{s}(H)\right|^{q}\left|D_{0}(H)\right|^{2}|P(H)|^{2} d H\right]^{1 / q} } \\
\leqslant & C \lim _{s \rightarrow \infty} s^{l / 2}\left[\int_{\zeta}\left|D_{0}(H) g_{s}(H) f(H)\right|^{p}\left|D_{0}(H)\right|^{2-p}|P(H)|^{2} d H\right]^{1 / p} \\
\cdot & {\left[\left.\int_{\zeta}|h(H)|^{q}\left|g_{s}(H)^{q}\right| D_{0}(H)\right|^{2}|P(H)|^{2} d H\right]^{1 / q} . }
\end{aligned}
$$

Now $g_{s}$ is positive; we apply Hölder again to bound the right side by 


$$
\begin{gathered}
\leqslant C \lim _{s \rightarrow \infty} s^{l / 2}\left\|g_{s}\right\|_{\infty}^{(p-1) / p}\left\|g_{s}\right\|_{\infty}^{(q-1) / q} . \\
{\left[\int_{\zeta}|f(\exp H)|^{p} g_{s}(H)\left|D_{0}(H)\right|^{2}|P(H)|^{2} d H\right]^{1 / p}} \\
\times\left[\int_{\zeta}|h(\exp H)|^{q} g_{s}(H)\left|D_{0}(H)\right|^{2}|P(H)|^{2} d H\right] .
\end{gathered}
$$

Now

$$
s^{l / 2}\left\|g_{s}\right\|_{\infty}^{1-1 / p}\left\|g_{s}\right\|_{\infty}^{1-1 / q}=s^{l / 2}\left\|g_{s}\right\|_{\infty}=\text { constant }
$$

Applying Lemma 3.4 completes the proof.

Theorem 3.7 provides a method for constructing multipliers on $G$; however, the hypothesis may be difficult to verify directly. Next we shall see that this verification reduces to Fourier multiplier problems on $\bar{G}$ and then often can be done easily. Thus we now develop a procedure for transplanting multiplier problems on $L_{\text {inv }}^{p}(g)$ to Fourier multiplier problems on $L^{p}(\xi)$. First we see how the Fourier transform is transplanted and then how multiplier operators are transplanted.

For $f$ a continuous compactly supported invariant function on $g$, the Fourier transform of $f$ was shown in [7, Theorem 3] to be given by

$$
\hat{f}\left(H_{2}\right)=\frac{P\left(H_{\rho}^{*}\right)}{|W| \overline{P\left(H_{2}\right)}} \int_{\zeta} P\left(H_{1}\right) f\left(H_{1}\right) \sum \epsilon(w) e^{-i B\left(w H_{1}, H_{2}\right)} d H_{1} .
$$

Since $f$ is $\operatorname{Ad} G$ invariant, it is $W$ invariant. The function $P$, however, is odd with respect to $W$, i.e., $P(w H)=\epsilon(w) P(H)$. Then $f p$ is odd, and, making a change of variables, we see that

$$
\hat{f}\left(H_{2}\right)=\frac{P\left(H_{\rho}^{*}\right)}{P\left(H_{2}\right)}[f P]^{\overline{2}}\left(H_{2}\right)
$$

where $\overline{ }$ denotes the Fourier transform on $\bar{G}$.

A similar computation for inverting the Fourier transform gives

$$
g^{\sim}(H)=\frac{P\left(H_{\rho}^{*}\right)}{P(H)}[\bar{P} g]^{\simeq}(H) \text { if } g=\hat{f} \text {. }
$$

If $K$ is a multiplier operator on $L_{\text {inv }}^{2}(g)$ with $(K f)^{\wedge}=m \hat{f}$, denote by $K_{0}$ the Fourier multiplier on $L^{2}(G)$ with $\left(K_{0} f\right)^{\bar{x}}=m \hat{f}$ : Then for $f \in L_{\text {inv }}^{2}(g) \cap$ $L_{\text {inv }}^{p}(g)$

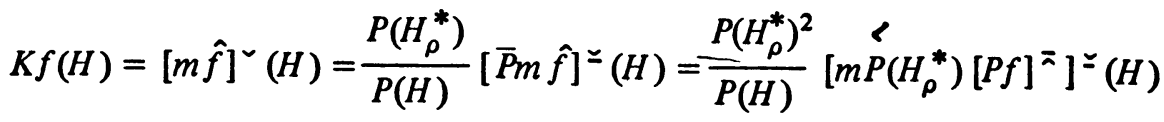

$$
\begin{aligned}
& =\frac{P\left(H_{\rho}^{*}\right)^{2}}{P(H)}\left[m[P f]^{\bar{\sim}}\right] \div(H)=\frac{P\left(H_{\rho}^{*}\right)^{2}}{P(H)} K_{0}(P f)(H) \text {. }
\end{aligned}
$$


PROPOSITION 3.8. If $m$ is a multiplier on $L^{p}(\xi)$ with respect to the weight function $|D(H)|^{2-p}$ and has operator norm $\|m\|$, then $m$ is a multiplier on $L_{\text {inv }}^{p}(g)$ with weight function $\left|D_{0}(H)\right|^{2-p}$ and operator norm at most $P\left(H_{\rho}^{*}\right)^{2}\|m\|$.

ProOF.

$$
\begin{aligned}
\int_{\mathfrak{\zeta}}|K f(H)|^{p}\left|D_{0}(H)\right|^{2-p}|P(H)|^{2} d H \\
\quad=P\left(H_{\rho}^{*}\right)^{2 p} \int_{\mathfrak{h}}\left|K_{0}(P f)(H)\right|^{p}\left|D_{0}(H)\right|^{2-p}|P(H)|^{2-p} d H \\
\quad \leqslant P\left(H_{\rho}^{*}\right)^{2 p}\|m\|^{p} \int_{h}|P f|^{p}|D(H)|^{2-p} d H \\
\quad=P\left(H_{\rho}^{*}\right)^{2 p}\|m\|^{p} \int_{\zeta}|f(H)|^{p}\left|D_{0}(H)\right|^{2-p}|P(H)|^{2} d H .
\end{aligned}
$$

4. Mean convergence. We come to the main result, a theorem concerning convergence in $L^{p}$ of the Fourier series expansion in characters of a central function on the compact semisimple Lie group $G$. Briefly this convergence question is handled as follows: convergence on the group reduces to uniform boundedness of the partial sum operators, which reduces by Theorem 3.7 to uniform boundedness of multiplier operators on $L_{\text {inv }}^{p}(g)$ with a weight function, which in turn reduces by Proposition 3.8 to the uniform boundedness of the multiplier operators on Euclidean space corresponding to a family of convex polygons. These last operators are to be bounded with respect to a weight function. Following an observation of Bochner [10], we simplify this question to one concerning uniform boundedness of the Hilbert transform with respect to a family of weight functions. Finally a theorem due to Hunt, Muckenhoupt and Wheeden [12] and a lemma due to Coifman allow us to establish this uniform boundedness. I am indebted to R. Coifman for pointing out the applicability of his lemma.

Typically the range of $p$ for which mean convergence holds is a small interval centered at $p=2$. We show that the Fourier transform maps $L_{\text {inv }}^{p}(g)$ into continuous functions for $p$ less than a critical exponent. Taken with a theorem of Strichartz or Theorem 2.5 this provides negative results for a range of $p$. Unfortunately for rank $G>1$, the mean convergence result together with this negative result do not exhaust all possible $p$ and so complete information on mean convergence is not yet available. An example with rank $G=2$ appears at the end of the section. I want to thank Peter Tomas for bringing my attention to Varopoulos's result on the Fourier transform on radial functions (see Stein and Weiss $[17$, p. 176]) which suggested this approach.

Before stating the main theorem, we shall specify the particular ways the partial sums of the Fourier series are formed.

Let $\omega_{1}, \ldots, \omega_{l}$ be the fundamental weights for $G$. The set $\left\{H_{\omega_{1}}^{*}, \ldots\right.$, $\left.H_{\omega_{l}}^{*}\right\}$ is a basis of $\mathscr{G}$ and the positive Weyl chamber, $\zeta^{+}$, is the set of all $H$ in $\mathscr{G}$ 
whose coordinates with respect to this basis are positive. Fix $H_{\nu}^{*}$ in $\overline{\bar{G}^{+}}$with $\left\|H_{\nu}^{*}\right\|=\left[-B\left(H_{\nu}^{*}, H_{\nu}^{*}\right)\right]^{1 / 2}=1$ and $-B\left(H_{\omega_{i}}^{*}, H_{\nu}^{*}\right)>0$ for $i=1, \ldots, l$. Let $\nu$ in $\left(\zeta^{\mathrm{C}}\right)^{\prime}$ be the element dual to $-i H_{\nu}^{*}$. If $f$ is in $L_{\text {inv }}^{2}(G) \cap L_{\text {inv }}^{p}(G)$ with Fourier series $\Sigma_{\lambda \in L} a_{\lambda} d_{\lambda} \chi_{\lambda}$, for each $R>0$ we define the partial sum operator

$$
S_{R} f=\sum_{\langle\lambda+\rho, \nu\rangle \leqslant R} a_{\lambda} d_{\lambda} \chi_{\lambda} \text {. }
$$

Let $r$ equal the number of positive roots $\alpha$ such that $\alpha\left(H_{\nu}^{*}\right) \neq 0$. Of course both $S_{R}$ and $r$ depend on $v$; however we shall suppress this in the notation.

THEOREM 4.1. If $f$ is in $L_{\text {inv }}^{p}(G)$ and $(2 r+1) /(r+1)<p<(2 r+1) / r$, then $S_{R} f$ converges to $f$ in the $L^{p}$ norm as $R \rightarrow \infty$.

The theorem will be proved in a series of steps.

Step 1. The conclusion is certainly true for trigonometric polynomials and these are dense in $L_{\text {inv }}^{p}(G)$. By standard functional analysis it is enough to show the operators $S_{R}$ are uniformly bounded:

Step 2. Reduction to a multiplier problem on $L_{\text {inv }}^{p}(g)$. We construct an invariant function $m$ on $g$ which when restricted to the lattice $\left\{H_{\lambda+\rho}^{*} \mid \lambda \in L\right\}$ gives the multiplier for $S_{R}$.

Set $\sigma=\left\{H \in\left\{\mid-B\left(H, H_{\nu}^{*}\right)=1\right\} ; \sigma\right.$ is a hyperplane intersecting the positive Weyl chamber. Set $E_{1}=\left\{H \in|5|-B\left(H, H_{\nu}^{*}\right) \leqslant 1\right\}$. Then $E_{1}$ is a half space containing zero and with boundary $\sigma$. For each $w \in W$ set $E_{w}=w E_{1}$. As $w$ acts by orthogonal transformations, $E_{w}=\left\{H \in \mathfrak{G} \mid-B\left(w^{-1} H, H_{\nu}^{*}\right)=\right.$ $\left.-B\left(H, w H_{\nu}^{*}\right) \leqslant 1\right\}$ and so is a half space. Set $C=\bigcap_{w \in W} E_{w}$ and set $m$ equal to the characteristic function of $C$. More generally, for each $R>0$ set $m_{R}$ equal to the characteristic function of $R C$.

LEMMA 4.2. (a) $R C \cap \mathrm{G}^{+}=R E_{1} \cap \mathrm{G}^{+}$.

(b) The sequence $\left\{m_{R}\left(H_{\lambda+\rho}^{*}\right)\right\}$ is the multiplier corresponding to $S_{R}$.

Proof. (a) It suffices to prove (a) with $R=1$. We always have $C \cap G^{+}$ contained in $E_{1} \cap G^{+}$so suppose $H$ is in $E_{1} \cap G^{+}$. Since both $H$ and $H_{\nu}^{*}$ are in $\overline{\zeta^{+}}, H=\Sigma h_{i} H_{\omega_{i}}^{*}$ with $h_{i}>0$ and $H_{\nu}^{*}=\Sigma c_{i} H_{\omega_{i}}^{*}$ with $c_{i} \geqslant 0$. For $w \in W$, $w H_{\omega_{i}}^{*}=-i w H_{\omega_{i}}=-i\left[H_{\omega_{i}}-\Sigma_{k=1}^{l} a_{i}^{k} H_{\alpha_{k}}\right]$ with the $a_{k}^{i}$ nonnegative integers and thi $\alpha_{k}$ the simple roots. Then $w H_{\omega_{i}}^{*}=H_{\omega_{i}}^{*}-\Sigma_{k=1}^{l} a_{k}^{i} H_{\alpha_{k}}^{*}$. We shall compute $-B\left(H, w H_{\nu}^{*}\right)$ and show that $H$ is in $E_{w}$.

$$
\begin{aligned}
-B\left(H, w H_{\nu}^{*}\right) & =-B\left(\sum h_{i} H_{\omega_{i}}^{*}, w \sum c_{j} H_{\omega_{j}}^{*}\right)=-\sum_{i, j} h_{i} c_{j} B\left(H_{\omega_{i}}^{*}, w H_{\omega_{j}}^{*}\right) \\
& =-\sum_{i, j} h_{i} c_{j} B\left(H_{\omega_{i}}^{*}, H_{\omega_{j}}^{*}\right)+\sum_{i, j} h_{i} c_{j} \sum_{k} a_{k}^{i} B\left(H_{\omega_{i}}^{*}, H_{\alpha_{k}}^{*}\right)
\end{aligned}
$$


But $h_{i}>0, c_{j} \geqslant 0, a_{k}^{i} \geqslant 0$ while $B\left(H_{\omega_{i}}^{*}, H_{\alpha_{k}}^{*}\right) \leqslant 0$, then

$$
-B\left(H, w H_{\nu}^{*}\right) \leqslant-\sum_{i, j} h_{i} c_{j} B\left(H_{\omega_{i}}^{*}, H_{\omega_{j}}^{*}\right)=-B\left(H, H_{\nu}^{*}\right) \leqslant 1 .
$$

(b) If $\lambda$ is in $L$, then $\langle\lambda+\rho, \nu\rangle \leqslant R$ if and only if $-B\left(H_{\lambda+\rho}^{*}, H_{\nu}^{*}\right) \leqslant R$ if and only if $H_{\lambda+\rho}^{*}$ is in $R E_{1} \cap \zeta^{+}$. By part (a) $H_{\lambda+\rho}^{*}$ is in $R C \cap \zeta^{+}$if and only if $H_{\lambda+\rho}^{*}$ is in $R E_{1} \cap \mathcal{S}^{+}$. Then we have the desired conclusion because the sequence $\left\{m_{R}\left(H_{\lambda+\rho}^{*}\right)\right\}$ contains precisely these lattice points.

In order to apply Theorem 3.7 to the multipliers $m_{R}$ it is necessary that they be continuous at $L$. We can arrange matters so that this condition is satisfied. For each $R>0$ the number of $\lambda$ in $L$ with $\langle\lambda+\rho, \nu\rangle \leqslant R$ is finite. Indeed if $\lambda=\Sigma l_{i} \omega_{i},\langle\lambda+\rho, \nu\rangle=\Sigma\left(l_{i}+1\right)\left\langle\omega_{i}, \nu\right\rangle$ and if this is $\leqslant R$ we must have $\left(l_{i}+1\right)\left\langle\omega_{i}, \nu\right\rangle \leqslant R$ for each $i=1, \ldots, l$. But $\nu$ was chosen so that $\left.\left\langle\omega_{i}, \nu\right\rangle\right\rangle 0$; then $\left(l_{i}+1\right) \leqslant R /\left\langle\omega_{i}, \nu\right\rangle$ and only finitely many nonnegative integers $l_{i}$ can satisfy this. Then it is clear that we can find an increasing sequence $R_{0}<R_{1}<R_{2} \cdots$ of positive numbers such that $m_{R_{i}}$ is continuous at $L$, $\langle\lambda+\rho, \nu\rangle \leqslant R_{0}$ if and only if $\lambda=0$, for $R_{i} \leqslant R<R_{i+1}$ the sets $\{\lambda \in L \mid\langle\lambda+$ $\left.\rho, \nu\rangle \leqslant R_{i}\right\}$ and $\{\lambda \in L \mid\langle\lambda+\rho, \nu\rangle \leqslant R\}$ are equal, and the set $\{\lambda \in L \mid\langle\lambda+$ $\left.\rho, \nu\rangle \leqslant R_{i}\right\}$ is not equal to $\left\{\lambda \in L \mid\langle\lambda+\rho, \nu\rangle \leqslant R_{i+1}\right\}$. Then to prove Theorem 4.1 it suffices to show the operators $S_{R_{i}}$ are uniformly bounded.

Let $K_{R_{i}}$ be the multiplier operators on Ad $G$ invariant functions on $g$ corresponding to $m_{R_{i}}$. By Theorem 3.7 the operators $S_{R_{i}}$ are uniformly bounded on $L_{\text {inv }}^{p}(G)$ if the operators $K_{R_{i}}$ are uniformly bounded on $L_{\text {inv }}^{p}(i)$ with the weight function $\left|D_{0}(H)\right|^{2-p}$.

Step 3. Reduction to Fourier multipliers on $L^{p}(\xi)$ with weight function. In fact by Proposition 3.8 the operators $K_{R_{i}}$ have the required properties if the functions $m_{R_{i}}$ are uniformly bounded Fourier multipliers on $L^{p}(\bar{G})$ with respect to the weight function $\mid D(H) i^{2-p}$.

Step 4. Reduction to Hilbert transform.

For $w \in W$ let $T_{w}$ be the Fourier multiplier operator on 5 corresponding to the multiplier $\chi_{w}=$ characteristic function of $E_{w}$. Let $K$ be the Fourier operator on $\mathfrak{G}$ corresponding to $m$. As $m=\Pi_{w \in W} \chi_{w}, K$ is the composition of the $T_{w}$. A sufficient condition for $K$ to be bounded on $L^{p}(\xi)$ with respect to a weight function is that each of the $T_{w}$ is so bounded.

It is useful to use different coordinates on $\bar{G}$ for each of the $T_{w}$. Let $\sigma_{0}$ be the $l-1$ dimensional subspace of 5 orthogonal to $\mathrm{R} H_{\nu}^{*}$ and let $\left\{H_{1}, \ldots\right.$, $\left.H_{l-1}\right\}$ be an orthonormal basis of $\sigma_{0}$. For notational convenience denote $H_{\nu}^{*}$ by $H_{l}$. For each $w$ in $W$ the set $\left\{w H_{1}, \ldots, w H_{l}\right\}$ is an orthonormal basis of $G$ and with these coordinates $\chi_{w}$ is the characteristic function of the half space 
$\left\{\left(h_{1}, \ldots, h_{l}\right) \mid h_{l} \leqslant 1\right\}$. Following Stein [16],

$$
\begin{aligned}
T_{w} f\left(h_{1}, \ldots, h_{l}\right) & =T_{w} f(\bar{h}) \\
& =\not / 2\left[f(\bar{h})+i e^{i B\left(\bar{h}, w H_{l}\right)} \bar{H}\left(e^{-i B\left(\cdot, w H_{l}\right)} f\right)(\bar{h})\right]
\end{aligned}
$$

where $\bar{H}$ is the classical Hilbert transform acting in the $l$ th coordinate. Then $T_{w}$ is bounded on $L^{p}(\xi)$ with a weight function if this is true for $\bar{H}$. Actually more can be said; if for $R>0$ one constructs the objects $T_{w}^{R}, \chi_{w}^{R}, m_{R}, K_{R}$ corresponding to $R E_{w}$, then $K_{R}$ is bounded on $L^{p}(\xi)$ with a weight function if each of the $T_{w}^{R}$ is. But

$$
T_{w}^{R} f(\bar{h})=1 / 2\left[f(\bar{h})+i e^{i B\left(\bar{h}, w R H_{l}\right)} \bar{H}\left(e^{-i B\left(\cdot, w R H_{l}\right)} f\right)(\bar{h})\right]
$$

and is bounded provided $\bar{H}$ is. Summarizing we have

LEMma 4.3. The functions $m_{R_{i}}$ define uniformly bounded Fourier multiplier operators on $L^{p}(\bar{G})$ with a weight function if the Hilbert transform $\bar{H}$ acting in the lth coordinate with respect to each of the bases $\left\{w H_{1}, \ldots, w H_{l}\right\}$ is bounded on $L^{p}(\xi)$ with the same weight function.

The weight function relevant to our problem is

$$
|D(H)|^{2-p}=\prod_{\alpha \in \Delta^{+}}|\sin \alpha(i H) / 2|^{2-p} .
$$

This weight function is a product so when computing the $L^{p}$ norm of $\bar{H} f$ only those factors that depend on the $l$ th coordinate are required. For $w \in W$ set $\Delta^{w}=\left\{\alpha \in \Delta^{+} \mid \alpha\left(w H_{l}\right) \neq 0\right\}$ and split $|D(H)|^{2-p}$ into two factors:

$$
\begin{aligned}
\left|D_{w}^{1}(H)\right|^{2-p} & =\prod_{\alpha \in \Delta^{w}}\left|\sin \frac{\alpha}{2}(i H)\right|^{2-p} \text { and } \\
\left|D_{w}^{2}(H)\right|^{2-p} & =\prod_{\alpha \in \Delta^{+} \backslash \Delta^{w}}\left|\sin \frac{\alpha}{2}(i H)\right|^{2-p} .
\end{aligned}
$$

Lemma 4.4. The cardinality of $\Delta^{w},\left|\Delta^{w}\right|$, is independent of $w$.

Proof. For $\beta \in \Delta$ set $|\beta|=\beta$ if $\beta>0$ and $-\beta$ if $\beta<0$. We show that $\Delta^{w}$ is in 1-1 correspondence with $\Delta^{1}$ where $\alpha \rightarrow\left|w^{-1} \alpha\right|$ is the bijection. It is well known that this map is 1-1. If $\alpha$ is in $\Delta^{w}$ then $\alpha\left(w H_{l}\right) \neq 0$ but this is the same as $w^{-1} \alpha\left(H_{l}\right) \neq 0$. Then $\left|w^{-1} \alpha\right|$ is in $\Delta^{1}$. Conversely if $\beta$ is in $\Delta^{1}, \beta\left(H_{l}\right)$ $\neq 0$ implies that $w \beta\left(w H_{l}\right) \neq 0$ so $|w \beta| \in \Delta^{w}$ and then the map is onto.

LEMMA 4.5. If for almost all $H_{0}$ in $w \sigma_{0}, \bar{H}$ is bounded on $L^{p}\left(\mathbf{R}^{1}\right)$ with the weight function $\left|D_{w}^{1}\left(H_{0}, h_{l}\right)\right|^{2-p}$ with norm $C$ independent of $H_{0}$, then $\bar{H}$ is bounded on $L^{p}(\xi)$ with weight function $|D(H)|^{2-p}$. 
ProOF.

$$
\begin{aligned}
\int_{\mathfrak{G}} \mid \bar{H} & \left.f(H)\right|^{p}|D(H)|^{2-p} d H \\
& =\int_{w \sigma_{0}}\left|D_{w}^{2}\left(H_{0}\right)\right|^{2-p}\left[\int_{\mathbf{R}^{1}}\left|\bar{H} f\left(H_{0}, h_{l}\right) i^{p}\right| D_{w}^{1}\left(H_{0}, h_{l}\right) i^{2-p} d h_{l}\right] d H_{0} \\
& \leqslant C \int_{w \sigma_{0}} \mid D_{w}^{2}\left(H_{0}\right) i^{2-p}\left[\int_{\mathbf{R}^{1}}\left|f\left(H_{0}, h_{l}\right) i^{p}\right| D_{w}^{1}\left(H_{0}, h_{l}\right) i^{2-p} d h_{l}\right] d H_{0} \\
& =C \int_{\zeta}|f(H)|^{p}|D(H)|^{2-p} d H .
\end{aligned}
$$

Fortunately the theory of the Hilbert transform with weight function has been completely worked out (see [12]). Suppose $\omega$ is a nonnegative function on $\mathbf{R}^{1}$. $\omega$ is said to satisfy condition $A_{p} \quad(1<p<\infty)$ if there is a constant $C$ such that for every interval $I$,

$$
\left[\int_{I} \omega(x) d x\right]\left[\int_{I} \omega(x)^{-1 /(p-1)} d x\right]^{p-1} \leqslant C|I|^{p} .
$$

The result is

Proposition 4.6. A necessary and sufficient condition for $\bar{H}$ to be bounded on $L^{p}\left(\mathbf{R}^{1}\right)$ with respect to $\omega$ is that $\omega$ satisfies condition $A_{p}$. The norm of $\bar{H}$ is dominated by the constant $C$ in condition $A_{p}$ times a function of $p$.

Step 5. Verification of condition $A_{p}$.

Our problem now appears to be computational. By Lemma 4.5 and Proposition 4.6 it is enough to determine for which $p$, if any, the collection of weight functions $\left\{\left|D_{w}^{1}\left(H_{0}, h\right)\right|^{2-p} \mid w \in W\right\}$ satisfy $A_{p}$ independent of $H_{0}$. For rank $G$ $=1$ this is the function $|\sin x|^{2-p}$ and it follows from a theorem of Hardy and Littlewood when the Hilbert transform is bounded for this weight.

We are grateful to R. Coifman for pointing out the next lemma which enables us to treat the general rank case.

LEMMA 4.7 (R. COIFMAN). Suppose $\omega_{1}, \omega_{2}$ are nonnegative functions on $\mathbf{R}^{1}$ both of which satisfy $A_{p}$ for some $p$ with constants $C_{1}, C_{2}$. Then for $0 \leqslant$ $a \leqslant 1, \omega_{a}=\omega_{1}^{a} \omega_{2}^{1-a}$ satisfies $A_{p}$ with constant $C_{1}^{a} C_{2}^{1-a}$.

Proof. Let $I$ be an interval and

$$
*=\left[\int_{I} \omega_{a}(x) d x\right]\left[\int_{I} \omega_{a}(x)^{-1 /(p-1)} d x\right]^{p-1}
$$

We use Hölder's inequality with $r=1 / a$ and $s=1 /(1-a)$.

and

$$
\int_{I} \omega_{a}(x) d x \leqslant\left[\int_{I} \omega_{1}(x) d x\right]^{a}\left[\int_{I} \omega_{2}(x) d x\right]^{1-a}
$$

$\int_{I} \omega_{a}(x)^{-1 /(p-1)} d x \leqslant\left[\int_{I} \omega_{1}(x)^{-1 /(p-1)} d x\right]^{a}\left[\int_{I} \omega_{2}(x)^{-1 /(p-1)} d x\right]^{1-a}$ 
Then

$$
* \leqslant C_{1}^{a} C_{2}^{1-a}|I|^{a p}|I|^{(1-a) p}=C_{1}^{a} C_{2}^{1-a}|I|^{p} .
$$

Corollary 4.7. If $\omega_{1}, \ldots, \omega_{s}$ satisfy $A_{p}$ and $a_{1}, \ldots, a_{s}$ are nonnegative numbers with $\Sigma a_{i}=1$, then $\omega_{1}^{a_{1}} \cdots \omega_{s}^{a}$ satisfy $A_{p}$.

We consider the weight function

$$
\left|D_{w}^{1}\left(H_{0}, h\right)\right|^{2-p}=\prod_{\alpha \in \Delta w}|\sin \alpha(i H) / 2|^{2-p} .
$$

By Lemma 4.3, $\left|\Delta^{w}\right|=\left|\Delta^{1}\right|=r$. Let $\omega_{i}(H)=\left|\sin \alpha_{i}(i H) / 2\right|^{r(2-p)}$ for the $\alpha_{1}, \ldots, \alpha_{r}$ in $\Delta^{w}$. If each $\omega_{i}$ satisfies $A_{p}$, then by Corollary $4.7\left|D_{w}^{1}(H)\right|^{2-p}$ $=\omega(H)=\Pi_{i=1}^{r} \omega_{i}(H)^{1 / r}$ also satisfies $A_{p}$. Using our coordinates $\left\{w H_{1}, \ldots\right.$, $\left.w H_{l}\right\}$, each of the $\omega_{i}$ is of the form $\left|\sin \left(c_{i} h+b_{i}\right)\right|^{r(2-p)}$. Condition $A_{p}$ is independent of translation and the scalar multiple $c_{i}$ is independent of $H_{0}$. Then $\omega_{i}\left(H_{0}, h\right)=\left|\sin \alpha_{i}(i H) / 2\right|^{r(2-p)}$ satisfies $A_{p}$ independent of $H_{0}$ if and only if $|\sin h|^{r(2-p)}$ does. It is known that the classical Hardy and Littlewood weight $|x|^{a}$ satisfies $A_{p}$ if and only if $-1<a<p-1$. If $a=r(2-p)$, solving for $p$ we have $(2 r+1) /(r+1)<p<(2 r+1) / r$. The following lemma will complete the proof of the theorem.

LEMMA 4.8. If $(2 r+1) /(r+1)<p<(2 r+1) / r,|\sin \alpha(H) / 2|^{r(2-p)}$ satisfies $A_{p}$ independent of $H_{0}$.

Proof. Using our convenient coordinates $|\sin \alpha(H) / 2|^{r(2-p)}=$ $|\sin (c h+b)|^{r(2-p)}$. Again the translation $b$ and frequency $c$ which is independent of $H_{0}$ will not affect the result, so we need only consider $|\sin h|^{r(2-p)}$. We verify $A_{p}$ for various types of intervals. Set $a=r(2-p)$.

(1) If valid for $|I|=\pi$, then valid for $|I|=k \pi$.

Proof. By periodicity

$$
\left[\int_{I}|\sin x|^{a} d x\right]\left[\int_{I}|\sin x|^{-a /(p-1)} d x\right]^{p-1} \leqslant c K^{p} \pi^{p}
$$

(2) If valid for $|I|=\pi$ then valid for $k \pi \leqslant|I| \leqslant(k+1) \pi, k \geqslant 1$.

Proof. $I=[a, b]$ and say $|I|=k \pi+\alpha$ with $0 \leqslant \alpha<\pi$.

$$
\begin{aligned}
{\left[\int_{I}|\sin x|^{a} d x\right] } & {\left[\int_{I}|\sin x|^{-a /(p-1)} d x\right]^{p-1} } \\
& \leqslant\left[\int_{a}^{b+\pi-\alpha}|\sin x|^{a} d x\right]\left[\int_{a}^{b+\pi-\alpha}|\sin x|^{-a /(p-1)} d x\right]^{p-1} \\
& \leqslant c[(k+1) \pi]^{p} .
\end{aligned}
$$

As $k \geqslant 1,(k+1) \pi \leqslant 2|I|$ so $A_{p}$ is verified with a new constant $2^{p} c$.

(3) Fix $\epsilon>0$. If $A_{p}$ valid for $|I|=\pi$ then valid for $\pi \geqslant|I|>\epsilon$.

Proof. $I=[a, b]$, 


$$
\begin{aligned}
& {\left[\int_{I}|\sin x|^{a} d x\right]\left[\int_{I}|\sin x|^{-a /(p-1)} d x\right]^{p-1}} \\
& \leqslant\left[\int_{a}^{b+\pi-|I|}|\sin x|^{a} d x\right]\left[\int_{a}^{b+\pi-|I|}|\sin x|^{-a /(p-1)}\right]^{p-1} \leqslant c \pi^{p} .
\end{aligned}
$$

Now $\pi=|I|+\pi-|I| \leqslant|I|+\pi-\epsilon=|I|+(\pi-\epsilon) \epsilon / \epsilon$. Set $R_{\epsilon}=(\pi-\epsilon) / \epsilon$, then $\pi \leqslant|I|+R_{\epsilon} \epsilon \leqslant\left(1+R_{\epsilon}\right)|I|$. So $A_{p}$ is verified with new constant $c\left(1+R_{\epsilon}\right)^{p} 2^{p}$.

(4) $|I|=\pi$. By periodicity it suffices to check for $[0, \pi]$. After the change of variable $\sin x=(1-t)^{1 / 2}$, the integrals become

$$
\left[\frac{1}{2} \int_{0}^{1}(1-t)^{(a-1) / 2} t^{-1 / 2} d t\right]\left[\frac{1}{2} \int_{0}^{1}(1-t)^{-(a-p+1) / 2(p-1)} t^{-1 / 2} d t\right]^{p-1} \text {. }
$$

These integrals are classical beta functions and are finite provided $(a-1) / 2>$ -1 and $(-a-p+1) / 2(p-1)>-1$. The first condition is always satisfied for $p<(2 r+1) / r$; while the second becomes on rearranging $a=r(2-p)<$ $p-1$ or $p>(2 r+1) /(r+1)$.

(5) $|I|<\epsilon$. If the distance of both endpoints of $I$ from the set of integer mutliples of $\pi$ is greater than $\epsilon$, use the estimate $\epsilon / 2 \leqslant|\sin x| \leqslant 1$. Condition $A_{p}$ is satisfied with possibly the new constant $2 / \epsilon$. Suppose the distance of either endpoint of $I$ from $\{Z \pi\}$ is less than $\epsilon$. We assume $3 \epsilon<\pi$; then $I$ is contained in an interval of the form $(k \pi-2 \epsilon, k \pi+2 \epsilon)$. Use the estimate $|x| / 2 \leqslant$ $|\sin x| \leqslant|x|$ and the integrals are bounded by

$$
\left[\int_{I}|x|^{a} d x\right]\left[\int_{I} 2^{a /(p-1)}|x|^{-a /(p-1)} d x\right]^{p-1} \leqslant 2^{a} c|I|^{p}
$$

provided $-1<a=r(2-p)<p-1$.

We come to a type of converse to Theorem 4.1. To the Lie algebra $g$ we associate a number $p_{c}$ with $1<p_{c}<2$ which we call the critical index. Its significance in analysis on $g$ is given by on $\mathrm{G}^{+}$.

Proposition 4.9. If $f$ is in $L_{\text {inv }}^{p}$ (g) with $1 \leqslant p<p_{c}$ then $\hat{f}$ is continuous

Let us defer the proof and see how this result yields negative results for mean convergence questions. We use the multipliers constructed at the beginning of this section. Suppose the operators $S_{R}, R>0$, were uniformly bounded operators on $L_{\text {inv }}^{p}(G)$. The function $m$ satisfies the hypotheses of Theorem 2.5 specialized to invariant functions or the hypotheses of Strichartz' theorem [18]. We would conclude then that $m$ was a multiplier on $L_{\text {inv }}^{p}(g)$. Applying Proposition 4.9 , it is necessary that $p>p_{c}$.

The proof of Proposition 4.9 involves a closer study of Bessel functions. We need a refinement of Harish-Chandra's result (Proposition 3.2) and this 
necessitates a digression into Lie algebra structure theory.

Let $\pi=$ set of simple roots $\alpha_{1}, \ldots, \alpha_{l}$, and let $S=$ set of nonempty subsets of $\pi$. If $S$ is in $S, S$ determines a root system $\Delta_{S} \subseteq \Delta$, namely, take $\Delta_{S}$ to be the totality of roots in $\Delta$ spanned by the elements of $S$. The Weyl group $W_{S}$ of $\Delta_{S}$ is generated by the reflections $s_{\alpha_{i}}, \alpha_{i} \in S$, and is a subgroup of $W$. The relevance of subsets $S$ is that they determine the walls and corners of the closed Weyl chamber, walls corresponding to one element set and corder $S$ with more than one element. The origin corresponds to $\pi$.

Fix $\epsilon>0$ and set $\mathscr{G}_{\epsilon}^{+}=\left\{H \in E_{,}^{+} \mid \alpha(H) \geqslant \epsilon\right.$ for all $\left.\alpha \in \Delta^{+}\right\}$and for each $S$ in $S$ set $\mathfrak{h}_{\epsilon, S}^{+}=\left\{H \in \mathfrak{G}^{+} \mid \alpha(H)<\epsilon\right.$ for all $\alpha \in S$ and $\beta(H) \geqslant \epsilon$ for all $\beta$ in $\pi \backslash S$ \}. Then $\mathfrak{G}^{+}$is the disjoint union of $\mathfrak{G}_{\epsilon}^{+}$and $\bigcup_{S \in S} \mathscr{G}_{\epsilon, S}^{+}$.

Recall that by Proposition 3.2,

$$
J\left(H_{\rho}^{*}, H\right)=D_{0}(H) \text { and } D_{0}(H)=c \prod_{\alpha \in \Delta^{+}} \frac{\sin \alpha(i H) / 2}{\alpha(H)} .
$$

On $\mathfrak{G}_{\epsilon, S}^{+}$, we have

$$
D_{0}(H)=c\left[\prod_{\alpha \in \Delta_{s}^{+}} \frac{\sin \alpha(i H) / 2}{\alpha(H)}\right]\left[\prod_{\alpha \in \Delta^{+} \backslash \Delta_{S}^{+}} \frac{\sin \alpha(i H) / 2}{\alpha(H)}\right]
$$

and for $\epsilon$ small the first factor is essentially constant. We shall see that all the Bessel functions behave like this.

We fix $S$ in $S$ and examine the behavior of $J\left(H, H^{\prime}\right)$ on $G_{\epsilon, S}^{+}$for $H, H^{\prime}$ in $\mathfrak{G}^{+}$and $H$ in $\mathfrak{G}_{\epsilon, S}^{+}$.

$$
J\left(H, H^{\prime}\right)=\frac{P\left(H_{\rho}^{*}\right)}{P\left(H^{\prime}\right) P(H)} \sum_{w \in W} \epsilon(w) e^{i B\left(w H, H^{\prime}\right) .}
$$

Define $P_{S}^{1}(H)$ and $P_{S}^{2}(H)$ by

$$
P_{S}^{1}(H)=\prod_{\alpha \in \Delta_{S}^{+}} \alpha(H) \text { and } P_{S}^{2}(H)=\prod_{\alpha \in \Delta_{S}^{+} \backslash \Delta_{S}^{+}} \alpha(H) .
$$

Since $P_{S}^{2}(H) J\left(H, H^{\prime}\right)$ is continuous on $\xi,\left(P\left(H_{\rho}^{*}\right) / P_{S}^{1}(H) \Sigma_{w \in W} \epsilon(w) e^{i B\left(w H, H^{\prime}\right)}\right.$ is continuous on $\mathfrak{h}$.

We shall use a coordinate system such that $H=H_{1}+H_{2}$ with $H_{1}$ is in the span of $\left\{H_{\alpha}^{*} \mid \alpha \in \Delta_{S}\right\}$ and $H_{2}$ is orthogonal to this space. Then

$$
\frac{P\left(H_{\rho}^{*}\right)}{P_{S}^{1}(H)} \sum_{w \in W} \epsilon(w) e^{i B\left(w H, H^{\prime}\right)}=\frac{P\left(H_{\rho}^{*}\right)}{P_{S}^{1}\left(H_{1}\right)} \sum \epsilon(w) e^{i B\left(w H_{1}, H^{\prime}\right)} e^{i B\left(w H_{2}, H^{\prime}\right)} .
$$

Since $H^{\prime}$ is in $\mathfrak{G}^{+}$, the function above is periodic in the $H_{2}$ variables. In fact, $H^{\prime}=\Sigma h_{i} H_{\omega_{i}}^{*}$ with $h_{i}>0$, so that if $\alpha$ is a simple root not in $S$,

$$
\begin{aligned}
B\left(w H_{2}+w\left(2 \pi\left(H_{\alpha}^{*} / h_{\alpha}\right)\right), H^{\prime}\right) & =B\left(w H_{2}, H^{\prime}\right)+\left(2 \pi / h_{\alpha}\right) B\left(w H_{\alpha}^{*}, H^{\prime}\right) \\
& =B\left(w H_{2}, H^{\prime}\right)+\left(2 \pi / h_{\alpha}\right) h_{\alpha} B\left(w H_{\alpha}^{*}, H_{\omega_{\alpha}}^{*}\right)
\end{aligned}
$$


and this equals $B\left(w H_{2}, H^{\prime}\right)+2 \pi k$ for some integer $k$. Since on $\xi_{\epsilon, S}^{+}$, the $H_{1}$ variables range over a compact set we must have $\mid\left(P\left(H_{\rho}^{*}\right) / P_{S}^{1}(H)\right) \Sigma \epsilon(w) e^{i B\left(w H, H^{\prime}\right)}$ bounded by a constant depending only on $H^{\prime}$. Then on $G_{\epsilon, S}^{+},\left|J\left(H, H^{\prime}\right)\right| \leqslant$ $C\left(H^{\prime}\right) / P\left(H^{\prime}\right) P_{S}^{2}(H)$. Motivated by this asymptotic behavior, we make the following definition.

Set $q_{c}$ equal to the infimum of $q \geqslant 2$ such that for each $S$ in $S$,

$$
\int_{\mathfrak{G}_{\epsilon, S}^{+}}\left|\frac{1}{P_{S}^{2}(H)}\right|^{q}|P(H)|^{2} d H<\infty
$$

and

$$
\int_{\zeta_{\epsilon}}+\left|\frac{1}{P(H)}\right|^{q}|P(H)|^{2} d H<\infty .
$$

Each of the integrands is the reciprocal of a nowhere vanishing polynomial and so $q_{c}$ is finite. We shall call the conjugate exponent $p_{c}\left(1 / q_{c}+1 / p_{c}=1\right)$ the critical exponent.

REMARK. We believe $q_{c}$ to be the $\inf _{q \geqslant 2}\left\{\left.q\left|\int_{\mathfrak{G}+}\right| D_{0}(H)\right|^{q}|P(H)|^{2} d H<\infty\right\}$; however we have been able to prove this only for a few cases.

We shall now prove Proposition 4.9.

Proof. For $H_{1}, H_{2}$ in $5^{+}$,

$$
\hat{f}\left(H_{1}\right)-\hat{f}\left(H_{2}\right)=\int_{\zeta^{+}} f(H) \overline{\left[J\left(H, H_{1}\right)\right.}-\left.\overline{\left.J\left(H, H_{2}\right)\right]} P(H)\right|^{2} d H .
$$

Applying Hölder's inequality, we obtain

$$
\left|\hat{f}\left(H_{1}\right)-\hat{f}\left(H_{2}\right)\right| \leqslant\|f\|_{p}\left\|J\left(\cdot, H_{1}\right)-J\left(\cdot, H_{2}\right)\right\|_{q} .
$$

We shall have proved the claim if we can justify applying the dominated convergence theorem to $\int_{\mathfrak{G}}+\left|J\left(H, H_{1}\right)-J\left(H, H_{2}\right)\right|^{q}|P(H)|^{2} d H$.

On each $\mathfrak{G}_{\epsilon, S}^{+}$and on a fixed compact neighborhood of $H_{2}$ for all $H$ in $\xi_{\epsilon, S}^{+}$and $H_{1}$ in this compact neighborhood we have the estimate $\left|J\left(H, H_{1}\right)\right| \leqslant$ $C\left(H_{2}\right) /\left|P\left(H_{1}\right)\right|\left|P_{S}^{2}(H)\right|$ so we can dominate $\left|J\left(H, H_{1}\right)-J\left(H, H_{2}\right)\right|$ by $M / P_{S}^{2}(H)$. Since $p<p_{c},\left|M / P_{S}^{2}(H)\right|^{q}$ is integrable.

We shall give two examples illustrating typical ranges of $p$ for which mean convergence results hold.

EXAMPLE 4.10. $G=S U(2), \operatorname{dim} \zeta=1, P(H)=h$ and computation gives $p_{c}=3 / 2$. There is only one way to sum the series and for that method $r=1$. Then $(2 r+1) /(r+1)=3 / 2$ and complete mean convergence information is obtained except at $p=3 / 2$. This is also a special case of Pollard's theorem.

Example 4.11. $G=S U(3), \operatorname{dim} \hbar=2$ and $\xi^{+}$is a $60^{\circ}$ cone. Take the the hyperplane $\sigma$ to be a line perpendicular to the $H_{\omega_{1}}^{*}$ axis. Using $H_{\omega_{1}}^{*}, H_{\omega_{2}}^{*}$ for coordinates, 


$$
D(H)=c \sin x \sin y \sin (x+y) .
$$

Then $r=2$ and $(2 r+1) /(r+1)=5 / 3$. The Euclidean multiplier $m$ is the characteristic function of the triangular region shown in Figure 1.

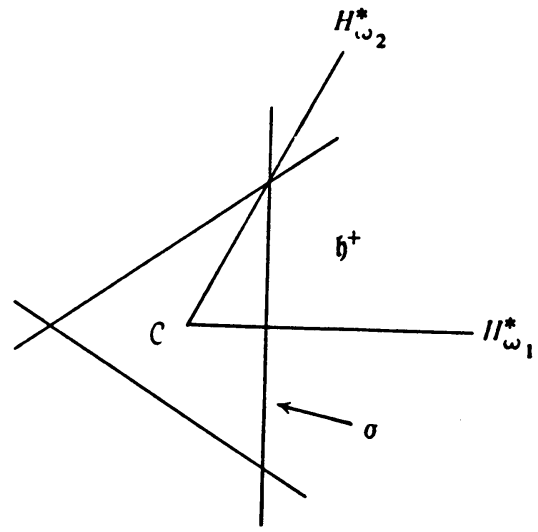

FIGURE 1

Suppose $\sigma$ was chosen to form an equilateral triangle with the $H_{\omega_{1}}^{*}$ and $H_{\omega_{2}}^{*}$ axes. Using $H_{\alpha+\beta}^{*}, H_{\alpha+\beta}^{* 1}$ for coordinates we get

$$
D(H)=c \sin x \sin (x-\sqrt{3} y) \sin (x+\sqrt{3} y) \text {. }
$$

Here $\sigma$ corresponds to the $y$ variable so $r=3$ and $(2 r+1) /(r+1)=7 / 4$. The Euclidean multiplier is the characteristic function of the hexagonal region shown in Figure 2.

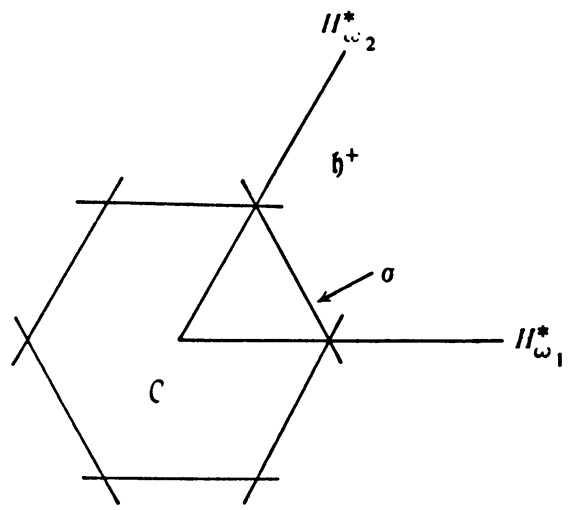

Figure 2

BIBLIOGRAPHY

1. N. Bourbaki, Éleménts de mathématique. Fasc. XXXIV. Groupes et algèbres de Lie. Chaps. IV-VI, Actualités Sci. Indust., no. 1337, Hermann, Paris, 1968. MR 39 \#1590.

2. Jean-Louis Clerc, Les sommes partielles de la décomposition en harmoniques sphériques ne convergent pas dans $L^{p}(p \neq 2)$, C. R. Acad. Sci. Paris Sér. A-B 274 (1972), A59-A61. MR 45 \#2405. 
2'. - Sommes de Riesz sur un groupe de Lie compact, C. R. Acad. Sci. Paris Sér. A-B 275 (1972), A591-A593. MR 46 \#5931.

3. R. Coifman and G. Weiss, Analyse harmonique noncommutative sur certaines espaces homogénes, Lecture Notes in Math., vol. 242, Springer-Verlag, Berlin, 1971.

4. - Central multiplier theorems for compact Lie groups, Bull. Amer. Math. Soc. 80 (1974), 124-126.

5. W. C. Connett and A. L. Schwartz, Weak type multipliers for Hankel transforms (preprint).

6. C. Fefferman, The multiplier problem for the ball, Ann. of Math. (2) 94 (1971), 330-336. MR 45 \#5661.

7. Harish-Chandra, Fourier transforms on a semisimple Lie algebra. I, Amer. J. Math. 79 (1957), 193-257. MR 19, 293.

8. - Spherical functions on a semisimple Lie group. I, Amer. J. Math. 80 (1958), 241-310. MR 20 \#925.

9. S. Helgason, Differential geometry and symmetric spaces, Pure and Appl. Math., Vol. 12, Academic Press, New York, 1962. MR 26 \#2986.

10. C. S. Herz, On the mean inversion of Fourier and Hankel transforms, Proc. Nat. Acad. Sci. U.S.A. 40 (1954), 996-999. MR 16, 127.

11. G. Hochschild, The structure of Lie groups, Holden-Day, San Francisco, Calif., 1965. MR 34 \#7696.

12. R. A. Hunt, B. Muckenhoupt and R. Wheeden, Weighted norm inequalities for the conjugate function and Hilbert transform, Trans. Amer. Math. Soc. 176 (1973), 227251. MR 47 \#701.

13. S. Igari, On the multipliers of Hankel transforms, Tôhoku Math. J. (2) 24 (1972), 201-206. MR 48 \#2684. \#5127.

14. K. deLeeuw, On $L_{p}$ multipliers, Ann. of Math. (2) 81 (1965), 364-379. MR 30

15. H. Pollard, The mean convergence of orthogonal series. III, Duke Math. J. 16 (1949), 189-191. MR 10, 450.

16. E. M. Stein, Singular integrals and differentiability properties of functions, Princeton Math. Ser., no. 30, Princeton Univ. Press, Princeton, N.J., 1970. MR 44 \#7280.

17. E. M. Stein and G. Weiss, Introduction to Fourier analysis on Euclidean spaces, Princeton Math. Ser., no. 32, Princeton Univ. Press, Princeton, N.J., 1971. MR 46 \#4102.

18. R. Strichartz, Multiplier transformations on compact Lie groups and Lie algebras (to appear).

DEPARTMENT OF MATHEMATICS, RICE UNIVERSITY, HOUSTON, TEXAS 77001 